Pacific Journal of Mathematic 


\title{
ON COMPACT METRIC SPACES WITH NONCOINCIDING TRANSFINITE DIMENSIONS
}

\author{
LEONID A. LUXEMBURG
}

For every no more than countable ordinal number $\alpha$ we shall define an ordinal number $\varphi(\alpha)$ such that for every compact metric space $X$ with ind $X \leq \alpha$ we have Ind $X \leq \varphi(\alpha)$ and there exists a compact metric spaces $X_{\alpha}$ with ind $X_{\alpha}=\alpha$, Ind $X_{\alpha}=\varphi(\alpha)$, where ind $X_{\alpha}$ and Ind $X_{\alpha}$ mean small and large transfinite inductive dimensions respectively. In particular we now extend the author's previous result on existence of compact metric spaces with noncoinciding transfinite dimensions.

1. Introduction. In this paper we consider only metric spaces. For instance, by a compact space we mean a compact metric space. All mappings we consider are continuous and $I^{n}$ denotes the $n$ dimensional euclidean cube.

1. Definitions and statements of main results.

DeFinition 1.1. (a) ind $X=-1 \Leftrightarrow X=\varnothing$.

(b) We assume that for every ordinal number $\alpha<\beta$ the class of spaces $X$ with ind $X \leqq \alpha$ is defined. Then, we say ind $X \leqq \beta$ if for every point $x \in X$ and a closed subset $F, x \notin F \subset X$, there exists a neighborhood $O x$ of $x$ such that:

$$
\begin{gathered}
O x \subset X \backslash F \\
\text { ind } \operatorname{Fr} O x \leqq \alpha<\beta^{1}
\end{gathered}
$$

We put ind $X=\min \{\beta$ : ind $X \leqq \beta\}$.

(c) We say that dimension $\operatorname{ind}_{x} X$ of a space $X$ in a point $x \in$ $X \leqq \beta$ if there exists such a base $\left\{O_{\lambda}: \lambda \in A\right\}$ at this point, so that

$$
\text { ind } \mathrm{FrO}_{\lambda}<\beta \text {. }
$$

We put $\operatorname{ind}_{x} X=\min \{\beta$ : ind $X \leqq \beta\}$.

Definition 1.2. (a) Ind $X=-1 \Leftrightarrow X=\varnothing$

(b) Let, for every ordinal number $\alpha<\beta$, the class of spaces $X$ with Ind $X \leqq \alpha$ be defined. Then, Ind $X \leqq \beta$ if for every pair of disjoint closed subsets $F$ and $G$ there exists a partition $C^{2}$

${ }^{1} \mathrm{Fr} A$ denotes the boundary of $A$.

${ }^{2}$ By a partition in $X$ between sets $A$ and $B$ we mean a closed set $C$ in $X$ such that $X \backslash C=U \cup V, U \cap V=\varnothing, A \subset U, B \subset V$ for some open sets $U$ and $V$ in $X$. 
between $F$ and $G$ such that Ind $C \leqq \alpha<\beta$. We put Ind $X=$ $\min \{\beta$ : Ind $X \leqq \beta\}$.

We note that we can also introduce the dimension ind using partitions, because if $x \in \bar{U} \subset X \backslash F$, and $U$ is open, then $\operatorname{Fr} U$ is a partition between $x$ and $F$. Obviously, ind $X \leqq$ Ind $X$. For spaces with a countable basis, in particular, for compact spaces dimension ind $X$ is no more than a countable ordinal (Hurewicz [4], p. 50), and Ind $X$ is no more than a countable ordinal even for all metric spaces (Smirnov [16], p. 418). Dimensions ind $X$ and Ind $X$ are not defined for every metric space. For example, the Hilbert cube $I^{\omega}$ does not have any transfinite dimension (Hurewicz [4], p. 51). Let

$$
Z=\bigcup_{n=1}^{\infty} I^{n}
$$

be the discrete union of cubes $I^{n}$. Then, obviously, ind $Z=\omega_{0}$. However, the dimension Ind $X$ doesn't exist. But if for a space $X$ the dimension Ind $X$ exists, then ind $X$ also exists. In this paper we solve the following problem: to find a function $\psi: \Omega \rightarrow \Omega$ defined on the set of all ordinal numbers $\alpha<\omega_{1}$ and satisfying the following conditions:

(i) for every compact space $X$, having dimension ind $X$, we have

$$
\psi(\text { Ind } X) \leqq \text { ind } X \leqq \operatorname{Ind} X \text {. }
$$

(ii) for every $\alpha<\omega_{1}$, there exists a compact space $X=X(\alpha)$ satisfying the following equalities:

$$
\text { Ind } X=\alpha ; \quad \text { ind } X=\psi(\alpha) \text {. }
$$

We shall also find such a function $\varphi: \Omega \rightarrow \Omega$, so that for every compact space $X$ with dimension ind $X$

(iii) ind $X \leqq$ Ind $X \leqq \varphi$ (ind $X$ ).

(iv) for every $\alpha<\omega_{1}$, there exists a compact space $X=X(\alpha)$ such that

$$
\text { ind } X=\alpha, \quad \text { Ind } X=\varphi(\alpha) \text {. }
$$

The first examples of compacta with noncoinciding transfinite dimensions were constructed by the author in [9]. Let us introduce some notations. In $\S 1$ small greek letters denote ordinal numbers. For every ordinal number $\beta$ the equality $\beta=\alpha+n$ holds, where $\alpha$ is a limit number or 0 , and $n=0,1,2, \cdots$. Then we set $K(\beta)=$ $n, J(\beta)=\alpha$. Further, for every $\beta \geqq \omega_{0}$ by $\tau(\beta)$ we denote an ordinal number, defined by the equality $\omega_{0}+\tau(\beta)=\beta$. If $\beta<\omega_{0}$ we set $\tau(\beta)=0$. 
Definition 1.3. Put $\varphi(\beta)=\omega_{0}+\omega_{0} \times \tau(\beta)$ for $\beta \geqq \omega_{0}$ and $\varphi(\beta)=\beta$ for $\beta<\omega_{0}^{3}$.

Numbers $\beta$ such that $\beta=\varphi(\beta)$ shall be called invariant. It is easy to prove that a number $\beta>\omega_{0}$ is invariant if and only if $\beta=$ $\omega_{0}^{\prime \prime \prime} \times \gamma^{4}$ for some $\gamma$; and, every $\alpha \leqq \omega_{0}$ is invariant.

Definition 1.4. Put $\psi(\beta)=\min \{\alpha: \varphi(\alpha) \geqq \beta\}$.

LEMMA 1.1. Functions $\varphi$ and is have the following properties:

(a) Let $\alpha=\omega_{0}+\beta+n$ where $\beta$ is a limit number or $0, n=$ $K(\alpha)$, and $\xi$ is a number such that $\omega_{0} \times \xi=\beta$. Then

$$
\psi(\alpha)= \begin{cases}\omega_{0}+\xi+1 & \text { if } n>0 \\ \omega_{0}+\xi & \text { if } n=0\end{cases}
$$

(b) $\psi(n)=\varphi(n)=n$ for $n=0,1,2 \cdots$.

(c) $\beta+\omega_{0} \geqq \varphi \circ \psi(\beta) \geqq \beta$.

(d) If $\alpha \geqq \omega_{0}$ then $\varphi(\alpha)$ is a limit number.

(e) If $\gamma>\beta$ then $\varphi(\gamma)>\varphi(\beta)$.

(f) Let $\gamma>\omega_{0}$, then $\psi(\gamma)=\psi(\beta)$ iff $J(\beta)=J(\gamma)$ and the numbers $K(\beta), K(\gamma)$ are either both equal to 0 , or both different from 0 .

(g) If $\beta$ is a nonlimit number, then $\psi(\beta)$ also is a nonlimit one.

(h) If $\gamma \leqq J(\alpha)<\alpha$ then $\psi(\gamma)<\psi(\alpha)$.

(i) If $\alpha$ is a limit number, then $\psi(\alpha)<\psi(\alpha+m)$ where $m=$ $1,2 \cdots$

(j) If $\alpha \geqq \beta$, then $\psi(\alpha) \geqq \psi(\beta)$.

(k) If $\alpha=\sup \left\{\gamma_{i}: i=1,2, \cdots\right\}$, then $\varphi(\alpha)=\sup \left\{\varphi\left(\gamma_{i}\right): i=\right.$ $1,2, \cdots\}$.

(l) If $\beta<\alpha$ and $\alpha$ is invariant number, then $\varphi(\beta)<\alpha$.

(m) If $\alpha=\sup \left\{\gamma_{i}: i=1,2, \cdots\right\}$, then $\psi(\alpha)=\sup \left\{\psi\left(\gamma_{i}\right): i=\right.$ $1,2, \cdots\}$.

(n) $\psi \circ \circ(\beta)=\beta$.

(o) $\varphi(\alpha)=\omega_{0} \times \alpha$ for $\alpha \geqq \omega_{0}^{2}, \varphi\left(\omega_{0}+p\right)=\omega_{0} \times(p+1), \varphi\left(\omega_{0} \times\right.$ $q+p)=\omega_{0}^{2} \times(q-1)+\omega_{0} \times p$ for $q=2,3, \cdots, p=0,1,2, \cdots$.

Proof. (a) Let $n>0$. Since $\varphi\left(\omega_{0}+\xi+1\right)=\omega_{0}+\omega_{0} \times \xi+\omega_{0}$ we have $\omega_{0}+\xi+1 \geqq \psi(\alpha)$. Further, $\varphi\left(\omega_{0}+\xi\right)=\omega_{0}+\omega_{0} \times \xi<\alpha$.

${ }^{3}$ We assume $0 \times \alpha=\alpha \times 0=0,0+\alpha=\alpha+0=\alpha$ for any $\alpha$.

Let $A, B$ be two well-ordered sets having types $\alpha, \beta$ respectively. In a product $A \times B$ we introduce the following well ordering: $(a, b)<\left(a^{\prime}, b\right)$ if $b^{\prime}>b$ or if $b^{\prime}=b$ and $a^{\prime}>a$. Then the type of $A \times B$ is denoted by $\alpha \times \beta$. Generally speaking $\alpha \times \beta \neq \beta \times \alpha$.

${ }^{4}$ By definition $\omega_{0}^{\omega_{0}}=\sup \left\{\omega_{0}^{n}: n=1,2, \cdots\right\}$ 
Consequently $\omega_{0}+\xi+1=\psi(\alpha)$. Now let $n=0$. Then $\varphi\left(\omega_{0}+\xi\right)=$ $\alpha$ and $\varphi\left(\omega_{0}+\xi^{\prime}\right)=\omega_{0}+\omega_{0} \times \xi^{\prime}<\alpha$ for $\xi^{\prime}<\xi$. Hence, $\psi(\alpha)=\omega_{0}+\xi$.

(b) is evident.

(c) follows from (a) and Definitions 1.3, 1.4.

(d), (e) are evident.

(f), (g), (h), (i) follow from (a).

(j) follows from (b) and Definition 1.4.

(k) If $\alpha<\omega_{0}$ then the assertion is evident. Let $\alpha=\omega_{0}+\beta$, then $\beta=\tau(\alpha)=\sup \left\{\tau\left(\gamma_{i}\right): i=1,2, \cdots\right\}$. Consequently, $\phi(\alpha)=$ $\sup \left\{\omega_{0}+\omega_{0} \times \tau\left(\gamma_{i}\right)=\varphi\left(\gamma_{i}\right): i=1,2 \cdots\right\}$.

(l) If $\alpha$ is invariant, then $\alpha=\varphi(\alpha)$ and $\varphi(\beta)<\varphi(\alpha)=\alpha$ by property (e).

(m) If $\alpha \leqq \omega_{0}$ or $\alpha$ is nonlimit number then the assertion is evident. Let $\alpha$ be a limit number $>\omega_{0}$. Then $\alpha=\omega_{0}+\omega_{0} \times \xi$ for some $\xi>0$. Then by virtue of (a), $\psi(\alpha)=\omega_{0}+\xi$. Obviously, for some $n>0$ each $\gamma_{k}(k \geqq n)$ has the representation:

$$
\gamma_{k}=\omega_{0}+\omega_{0} \times \xi_{k}+K\left(\gamma_{k}\right)
$$

and $\gamma_{k}<\omega_{0}$ for $k<n$. Let $\xi$ be a limit number and $\xi>\sup _{i>n}\left\{\xi_{i}\right\}$. Then $\sup \left\{\xi_{i}\right\}+1<\xi$ and $\sup \left\{\gamma_{i}\right\} \leqq \sup \left\{\omega_{0}+\omega_{0} \times \xi_{i}+\omega_{0}\right\}<\omega_{0}+\omega_{0} \times \xi=\alpha$ which contradicts the condition. Therefore

$$
\xi=\sup \left\{\xi_{i}: i>n\right\} \text {. }
$$

Consequently, by virtue of (a), (1) and (2)

$$
\psi(\alpha)=\omega_{0}+\xi=\sup \left\{\omega_{0}+\xi_{i}: i>n\right\} \leqq \sup \left\{\psi\left(\gamma_{i}\right): i=1,2 \cdots\right\} .
$$

The inequality $\psi(\alpha) \geqq \sup \left\{\psi\left(\gamma_{i}\right): i>n\right\}$ follows from (j). If $\xi=\xi_{0}+$ 1 , then $\alpha=\omega_{0}+\omega_{0} \times \xi_{0}+\omega_{0}$ and there is a number $\gamma_{i}$ such that $\gamma_{i} \geqq \omega_{0}+\omega_{0} \times \xi_{0}+1$. By virtue of (a) $\psi\left(\gamma_{i}\right)=\omega_{0}+\xi_{0}+1=\omega_{0}+$ $\xi=\psi(\alpha)$. Hence $(\mathrm{m})$ is proved.

(n) follows from (l), (o) is evident.

We shall use the addition theorem for inductive dimensions, proved in [8], Levsenko, Theorem 1, p. 255 and Theorem 1, p. 257. Let $\alpha, \beta$ be limit numbers and $p, q$ integers $\geqq 0$. Then put:

$$
k(\alpha, p, \beta, q)= \begin{cases}\alpha+p & \text { for } \beta<\alpha \\ \beta+q & \text { for } \alpha<\beta \\ \alpha+p+q+1 & \text { for } \alpha=\beta\end{cases}
$$

AdDition THEOREM (L). Let the hereditarily normal space $R$ be a union of two closed sets $R_{1}$ and $R_{2}$, having dimensions ind $R_{1} \leqq$ $\alpha+p$ (respectively Ind $R_{1} \leqq \alpha+p$ ) and ind $R_{2} \leqq \beta+q$ (respectively 
Ind $R_{2} \leqq \beta+q$ ). Then $R$ has dimension ind $R$ (respectively Ind $R$ ) and the following inequality holds:

$$
\text { ind } R(\text { Ind } R) \leqq k(\alpha, p, \beta, q) \text {. }
$$

THEOREM $^{5}$ 1.1. Let $X$ be a completely normal bicompactum (not necessarily metrizable) having dimension ind $X$. Then $X$ has dimension Ind $X$ and

$$
\text { Ind } X \leqq \varphi(\text { ind } X)
$$

Proof. If ind $X<\omega_{0}$ it is well known (Vedenisov [19] that ind $X=$ Ind $X=\varphi$ (ind $X$ ). Suppose that ind $X=\beta \geqq \omega_{0}$ and for all $\gamma<\beta$ and for any completely normal bicompactum $X$ having ind $X=\gamma$ the theorem is proved. Let $F$ and $G$ be closed disjoint subsets of $X$. Since $X$ is a bicompactum, there exists a finite collection of open sets $O_{1}, \cdots, O_{s}$ in $X$, such that:

$$
\begin{gathered}
\bar{O}_{i} \cap G=\varnothing \text {, ind } \operatorname{FrO}_{i} \leqq \gamma_{i}<\beta, \cup\left\{O_{i}: i=1, \cdots, s\right\} \supset F, \\
(i=1, \cdots, s) .
\end{gathered}
$$

By the inductive assumption dimensions Ind $\mathrm{FrO}_{i}$ exist and

$$
\text { Ind } \mathrm{FrO}_{i} \leqq \varphi \text { (ind } \mathrm{FrO}_{i} \text { ). }
$$

By property (e) from Lemma 1.1, $\varphi$ (ind $\left.F r O_{i}\right)<\varphi($ ind $X$ ). Since by property (d) from Lemma $1.1 \varphi(\beta)$ is a limit number,

$$
\varphi\left(\text { ind } \mathrm{FrO}_{i}\right)+\omega_{0} \leqq \varphi(\beta) \text {. }
$$

From Theorem L and (3) it follows that

$$
\begin{aligned}
& \text { Ind }\left(\cup\left\{F r O_{i}: i=1, \cdots, s\right\}\right) \leqq \\
&+\sum_{i=1}^{s} K\left(\operatorname{Ind} F r O_{i}\right)+(s-1) \\
&<\max \left\{\varphi\left(\text { ind } F r O_{i}\right): i=1, \cdots, s\right\}+\omega_{0} \leqq \varphi(\beta) .
\end{aligned}
$$

Since the set $\cup\left\{F_{r} O_{i}: i=1, \cdots, s\right\}$ obviously contains a partition between $F$ and $G$, we have

$$
\text { Ind } C \leqq \text { Ind } \bigcup_{i=1}^{s} F r O_{i}<\varphi(\beta) \text {. }
$$

COROLlARY 1.1. For any completely normal bicompactum (not necessarily metrizable) $X$ having dimension ind $X$, we have Ind $X \geqq$ ind $X \geqq \psi($ Ind $X)$.

${ }^{5}$ In [8] Theorem 2, p. 260 an upper bound for dimension Ind was also obtained, however it is less exact for completely normal bicompacta. 
Proof. Let us suppose that ind $X<\psi($ Ind $X)$; then by definition of the function $\psi, \varphi($ ind $X)<$ Ind $X$ which contradicts Theorem 1.1 .

COROLLARY 1.2. Let $X$ be a completely normal bicompactum (not necessarily metrizable). Then

(a) if ind $X$ is an invariant number we have ind $X=$ Ind $X$.

Let $\alpha$ be an invariant number, then

(b) If Ind $X=\alpha+1$, then ind $X=$ Ind $X$.

(c) If Ind $X=\alpha$, then ind $X=$ Ind $X$.

Proof. (a) follows from Theorem 1.1.

(b) If ind $X \leqq \alpha$, then $\varphi$ (ind $X) \leqq \varphi(\alpha)=\alpha$, by property (e) of Lemma 1.1, and consequently, by Theorem 1.1 Ind $X \leqq \alpha$, which contradicts our condition. Therefore, ind $X=\alpha+1$.

(c) If ind $X<\alpha$, then by virtue of property (e) of Lemma 1.1, $\varphi($ ind $X)<\alpha$. Then by Theorem 1.1 Ind $X<\alpha$, which contradicts the condition. Hence ind $X=\alpha$.

THEOREM 1.2. For any countable ordinal number $\beta<\omega_{1}$, there exists a weakly countable dimensional ${ }^{6}$ compactum $X_{\beta}$, such that Ind $X_{\beta}=\beta$, ind $X_{\beta}=\psi(\beta)$.

TheOREM 1.2'. For any ordinal number $\beta<\omega_{1}$, there exists a weakly-countable dimensional compactum $Y_{\beta}$ such that Ind $Y_{\beta}=\varphi(\beta)$, ind $Y_{\beta}=\beta$.

Theorem 1.2' follows [from Theorem 1.2, since $\psi \circ \varphi(\beta)=\beta$ by Lemma $1.1(\mathrm{n})$. We can set in Theorem 1.2 $Y_{\beta}=X_{\varphi(\beta)}$. Therefore, we shall prove only Theorem 1.2.

Theorem 1.2 and Corollary 1.1 show that the function $\psi$ possesses properties (i) and (ii). Theorem 1.2' and Theorem 1.1 show that the function $\phi$ possesses properties (iii), (iv). We restrict our investigation to the field of compact spaces because every separable space $X$ is contained in a compactum $K$ such that ind $K=$ ind $X$; Ind $K=$ Ind $X$ (see [10], Luxemburg).

Problem. Let $\alpha, \beta$ be two ordinal numbers. Under what conditions does there exist a compactum $X$ such that

$$
\text { ind } X=\alpha \text {, Ind } X=\beta \text { ? }
$$

${ }^{B}$ A space is called weakly countable dimensional if it is a union of a countable number of its closed finite dimensional subsets. In this work by finite dimensional space we mean a space with finite dimension dim. 
From Theorem 1.1 it follows that the condition

$$
\alpha \leqq \beta \leqq \varphi(\alpha)<\omega_{1} .
$$

is necessary. Is this condition sufficient? For this it is necessary and sufficient to prove that for any $\alpha<\omega_{1}$ there exists a compactum $Y_{\alpha}$ such that

$$
\text { Ind } Y_{\alpha}=\text { ind } Y_{\alpha}=\alpha \text {. }
$$

Indeed, by Theorem 1.2 there exists a compact $Z$ such that

$$
\text { Ind } Z=\beta \text {, ind } Z=\psi(\beta) \text {. }
$$

Let $\alpha$ satisfy the condition (5), then by properties (n), (j) of Lemma 1.1

$$
\psi(\beta) \leqq \psi \circ \varphi(\alpha)=\alpha .
$$

Put $X=Y_{\alpha} \cup Z, Y_{\alpha} \cap Z=\varnothing$. Then, by virtue of (6), (7) and (8), the condition (4) holds.

We begin now to prove preliminary results for Theorem 1.2.

\section{Systems of general position.}

Definition 2.1. A system of finite dimensional sets $A=\left\{A_{\mu}\right.$ : $\mu \in \mathscr{M}\}$ is in general position (g.p.) if for any finite number of indexes $\mu_{(1)}, \cdots, \mu_{(k)}$ of $\mathscr{t}$ we have either

$$
\operatorname{dim} \cap\left\{A \mu_{(i)}: i=1, \cdots, k\right\} \leqq \max \left\{\operatorname{dim} A \mu_{(i)}: i=1, \cdots, k\right\}-(k-1)
$$

or

$$
\cap\left\{A \mu_{(i)}: i=1, \cdots, k\right\}=\varnothing .
$$

We shall write $A$ is (g. p.) if $A$ is in general position.

In this section we consider $A=\left\{A_{\mu}: \mu \in \mathscr{C}\right\}$ to be a locally countable system of closed sets in a finite dimensional space $X$, such that $X \in A$.

Lemma 2.1. Let $F=\left\{F_{\nu}: \nu \in \mathscr{N}\right\}$ be a locally countable system of closed sets in $X$, such that for every $\nu \in \mathscr{N}$ the system $A(\nu)=A \cup$ $\left\{F_{\nu}\right\}$ is g. p. Then, for the set $\varphi=\cup\left\{F_{\nu}: \nu \in \mathscr{N}\right\}$ the system $B=$ $A \cup\{\varphi\}$ is g.p.

Proof. Let $A \mu_{(1)}, \cdots, A \mu_{(k)}$ be a finite subsystem of $A$. Since $A(\nu)$ is g.p., for every $\nu \in \mathscr{N}$ we have

$$
\begin{gathered}
\operatorname{dim}\left(\cap\left\{A \mu_{(i)}: i=1, \cdots, k\right\} \cap F_{\nu}\right) \leqq \max \left\{\operatorname{dim} A \mu_{(i)}, \operatorname{dim} F_{\nu}:\right. \\
i=1, \cdots, k\}-k
\end{gathered}
$$


if $F_{\nu} \cap \cap\left\{A \mu_{(i)}: i=1, \cdots, k\right\} \neq \varnothing$. Since

$$
\cap\left\{A \mu_{(i)}: i=1, \cdots, k\right\} \cap \varphi=\cup\left\{\cap\left\{A \mu_{(i)}: i=1, \cdots, k\right\} \cap F_{\nu}: \nu \in \mathscr{N}\right\}
$$

and the closed sets

$$
G_{\nu}=F_{\nu} \cap \cap\left\{A \mu_{(i)}: i=1, \cdots, k\right\}, \nu \in \mathscr{N}
$$

form a locally countable system, then by virtue of the sum-theorem for $\operatorname{dim}$ we have:

$$
\begin{aligned}
& \operatorname{dim}\left\{\cap\left\{A \mu_{(i)}: i=1, \cdots, k\right\} \cap \varphi\right\} \leqq \sup \left\{\operatorname { d i m } \left\{\cap\left\{A \mu_{(i)}: i=1, \cdots, k\right\}\right.\right. \\
& \left.\left.\cap F_{\nu}\right\}: \nu \in \mathscr{N}\right\} \\
& \leqq \sup \left\{\operatorname { m a x } \left\{\operatorname{dim} A \mu_{(i)}, \operatorname{dim} F_{\nu}: \nu \in \mathscr{N}, \cap\left\{A \mu_{(i)}: i=1, \cdots, k\right\}\right.\right. \\
& \left.\left.\cap F_{\nu} \neq \varnothing\right\}-k\right\} \\
& \leqq \max \left\{\operatorname{dim} A \mu_{(i)}, \operatorname{dim} \varphi: i=1, \cdots, k\right\}-k
\end{aligned}
$$

if $\varphi \cap \cap\left\{A \mu_{i}^{\prime}: i=1, \cdots, k\right\} \neq \varnothing$.

Lemma 2.2. Let $F \subset U \subset X$, where $F$ is closed and $U$ is open in $X$, and let $A$ be a locally countable system of sets such that $A$ is g.p. If $U$ intersects no more than a countable number of elements of the system $A$, then there exists an open set $W$, such that

$$
F \subset W \subset \bar{W} \subset U
$$

and

$$
\text { the system } A \cup\{F r W\} \text { is g.p. }
$$

Proof. Let $C$ be a subsystem of the system $A$, consisting of all sets intersecting $U$, and let $D$ be a system consisting of all intersections of finite collection sets in $C$. Since, by hypothesis, the system $C$ is no more than countable, the system $D$ is also no more than countable. Then, see [14] Morita, there exists an open set $W$ such that condition (1) holds and

$$
\operatorname{dim}(F r W \cap L) \leqq \operatorname{dim} L-1 \text { for } L \in D .
$$

Let $A \mu_{(1)}, \cdots, A \mu_{(k)}$ be a finite collection of elements of $A$ such that $L=\cap\left\{A \mu_{(i)}: i=1, \cdots, k\right\}$. Then, from property (3), it follows that

$$
\begin{gathered}
\operatorname{dim} F r W \cap \cap\left\{A \mu_{(i)}: i=1, \cdots, k\right\} \leqq \operatorname{dim} \cap\left\{A \mu_{(i)}: i=1, \cdots, k\right\}-1 \\
\leqq \max \left\{\operatorname{dim} F r W, \operatorname{dim} A \mu_{(i)}: i=1, \cdots, k\right\}-k .
\end{gathered}
$$

Lemma 2.3. The assertion of Lemma 2.2 is true without the assumption that $U$ intersects no more than a countable number of elements of the system $A$. 
Proof. Let $\mathscr{Q}=\left\{U_{\beta}: \beta \in B\right\}$ be a locally finite open covering of $X$, such that every element $U_{\beta} \in U$ intersects no more than a countable number of elements of $A$ and

$$
\text { if } U_{\beta} \cap F \neq \varnothing \text { then } U_{\beta} \subset U, \beta \in B \text {. }
$$

Let $F=\left\{F_{\beta}: \beta \in B\right\}$ be a combinatorial refinement ${ }^{7}$ of the covering $\mathscr{C}$, and let $F_{\beta}$ be closed for every $\beta \in B$. Then, by Lemma 2.2 for any $\beta \in B$ there exists a set $W_{\beta}$ such that

$$
F_{\beta} \subset W_{\beta} \subset \bar{W}_{\beta} \subset U_{\beta}
$$

and the system

$$
A(\beta)=A \cup\left\{F r W_{\beta}\right\} \text { is g.p. }
$$

We set $W=\cup\left\{W_{\beta}: W_{\beta} \cap F \neq \varnothing ; \beta \in B\right\}$. From properties (5), (6) it follows that $F \subset W \subset \bar{W} \subset U$. Since $\mathscr{C}$ is locally finite and (5) is true it follows that

$$
\operatorname{Fr} W \subset H=\cup\left\{F r W_{\beta}: \beta \in B\right\} .
$$

From Lemma 2.1 and (6) it follows that the system $A \cup\{H\}$ is g.p. Consequently, $A \cup\{F r W\}$ is g.p.

Proposition 2.1. Let $U$ be an open covering of a space $X$. Then there exists a locally finite open covering $W=\left\{W_{\nu}: \nu \in N\right\}$ of $X$, which is a refinement of $U$, and such that

(7) The system $B=A \cup\left\{F r W_{\nu}: \nu \in \mathscr{N}\right\}$ is g.p.

(8) $\operatorname{dim} F r W_{\nu}<\operatorname{dim} X$.

Proof. Let $V=\left\{V_{\nu}: \nu \in \mathscr{N}\right\}$ be a locally finite open refinement of $U$, and $F=\left\{F_{\nu}: \nu \in \mathscr{N}\right\}$ be a combinatorial closed refinement of $V$. We can suppose that the set of indexes $\mathscr{N}$ is well ordered. We shall construct by induction open sets $W_{\nu}$ such that properties (7), (8) hold and

$$
F_{\nu} \subset W_{\nu} \subset \bar{W}_{\nu} \subset V_{\nu} \text { for } \nu \in \mathscr{N} \text {. }
$$

Let $\nu=0$. Then by Lemma 2.3 there exists an open set $W_{0}$ satisfying condition (2) for $W=W_{0}$ and condition (9) for $\nu=0$. Suppose that for all $\nu<\nu_{0}$ we have constructed sets $W_{\nu}$ satisfying condition (9) and such that the system $A(\nu)=A \cup\left\{F r W_{\nu^{\prime}}: \nu^{\prime}<\nu\right\}$ is g.p. Then, obviously, the system

$$
C=A \cup\left\{F r W_{\nu}: \nu<\nu_{0}\right\} \quad \text { is g.p. }
$$

${ }^{7}$ i.e. $F_{\beta} \subset U_{\beta}$ for every $\beta \in B$. 
Since $A$ is locally countable and $W$ is a locally finite system, $C$ is locally countable. By Lemma 2.3 (we now set $A=C$ ) there exists an open set $W_{\nu_{0}}$ such that the system $A \cup\left\{F r W_{\nu}: \nu \leqq \nu_{0}\right\}$ is g.p. Hence, sets $W_{\nu}$ satisfying condition (7) have been constructed. Since $X \in A$, condition (8) follows from (7).

CoROLLARY 2.1. Let $\mathscr{U}_{i}, i=1,2, \cdots$, be a countable collection of open coverings of $X$. Then there exist locally finite open coverings $\mathscr{V}_{i}=\left\{V_{\mu}^{i}: \mu \in \mathscr{M}\right\}$ such that:

(i) $\mathscr{\mathscr { T }}_{i}$ is a refinement of $\mathscr{Q}_{i}$

(ii) The system $A \cup\left\{F r V_{\mu}^{i}: i=1,2, \cdots, \mu \in \mathscr{C}\right\}$ is g.p.

Proof. We can construct the coverings $\mathscr{\mathscr { T }} i$ by induction, using Proposition 2.1.

CoROLLARY 2.2. Every finite dimensional space $X$ has $a$ locally finite open basis with boundaries in general position and having dimension $<\operatorname{dim} X$.

Proof. Let $\mathscr{U}_{i}$ be a covering of $\operatorname{mesh} \mathscr{U}_{i}^{8}<1 / i$. Then we obtain our assertion using Corollary 2.1.

We denote by $d_{k}(X)$ the greatest lower bound of all numbers $\varepsilon$ such that there exists an open covering $U(\varepsilon)$ of $X$ with order $U(\varepsilon) \leqq k$ and mesh $U(\varepsilon)<\varepsilon$. The number $d_{k}(X)$ is called the $k$ thcoefficient of Urysohn of $X$.

CoROLlary 2.3. Let $\left\{\mathscr{U}_{i}: i=1,2, \cdots\right\}$ be a countable collection of coverings of $X$ and let $\left\{\varepsilon_{i}\right\}$ be a sequence of positive numbers. Then there exist closed sets $C_{i} \subset X$ such that

(i) the system $A \cup\left\{C_{i}: i=1,2, \cdots\right\}$ is g.p.

(ii) the set $X \backslash C_{i}$ is a union of disjoint open sets with diameter $<\varepsilon_{i}$ such that each of them is contained in some set $U_{\alpha} \in \mathscr{U}_{i}$.

(iii) $\operatorname{dim} C_{i} \leqq \operatorname{dim} X-1$.

(iv) $d_{1}\left(X \backslash C_{i}\right)<\varepsilon_{i}$.

Proof. Let $\mathscr{W}_{i}$ be an open refinement of $\mathscr{W}_{i}$ and let mesh $\mathscr{W}_{i}<\varepsilon_{i} / 2$, then by Corollary 2.1 there exist locally finite open coverings of $X \mathscr{V}_{i}=\left\{V_{\nu}^{i}: \nu \in \mathscr{N}_{i}\right\}$ such that:

$$
\begin{array}{r}
\mathscr{W}_{i} \text { is a refinement of } \mathscr{W}_{i} \text { and } \mathscr{U}_{i}, \text { mesh } \mathscr{V}_{i}<\varepsilon_{i} / 2, \\
\text { system } B=A \cup\left\{F r V_{\nu}^{i}: \nu \in \mathscr{N}_{i}, i=1,2, \cdots\right\}
\end{array}
$$

is g.p. Put

\footnotetext{
${ }^{8}$ i.e., diameter $U<1 / i$ for any $U \in \mathscr{U}_{i}$
} 


$$
C_{i}=\cup\left\{F r V_{\nu}^{i}: \nu \in \mathscr{N}\right\} .
$$

Consequently applying Lemma 2.1 . we obtain that the system $A \cup$ $\left\{C_{i}: i \leqq k\right\}$ is g.p. for each $k=1,2, \cdots$. Therefore, property (i) also holds. Property (ii) follows from (10), (11). Then, property (iii) follows from (i), because $X \in A$, and $X \cap C_{i}=C_{i}$. Property (iv) follows from (ii).

LEMma 2.4. Let $F=\left\{F_{\mu}: \mu \in \mathscr{C}\right\}$ be a locally finite collection of closed sets in space $X$ and let $\mathscr{C}=\left\{U_{\mu}: \mu \in \mathscr{M}\right\}$ be a collection of open sets, such that

(i) $U_{\mu} \supseteqq F_{\mu}$ for any $\mu \in \mathscr{M}$.

Then there exists a collection $\mathscr{W}=\left\{W_{\mu}: \mu \in \mathscr{M}\right\}$ of open sets such that

(ii) $F_{\mu} \subset W_{\mu} \subset \bar{W}_{\mu} \subset U_{\mu}(\mu \in \mathscr{C})$.

(iii) If $\cap\left\{F \mu_{(i)}: i=1, \cdots, k\right\}=\varnothing$ then $\cap\left\{W_{\mu_{(i)}}: i=1, \cdots, k\right\}=$ $\varnothing(k=1,2, \cdots)$.

Proof. Since a collection $F$ is locally finite, we can select for every point $x \in X$ an open set $O_{x} \ni x$ such that:

$$
\text { If } x \notin F \mu \text {, then } O x \cap F \mu=\varnothing \text {. }
$$

Since $X$ is a metric space and, consequently, paracompact, we can find an open covering $\mathscr{V}$ of a space $X$ such that $\mathscr{V}$ is a star refinement of the covering $\{O x: x \in X\}$. We consider a system of sets: $\left\{V_{\mu}=S t\left(F_{\mu}, \mathscr{V}\right): \mu \in \mathscr{M}\right\}^{9}$. We shall prove that

$$
\begin{aligned}
& \text { If } \cap\left\{F_{\mu(i)}: i=1, \cdots, k\right\}=\varnothing \text { then } \cap\left\{V_{\mu_{(i)}}: i=1, \cdots, k\right\} \text {, } \\
& (k=1,2, \cdots) \text {. }
\end{aligned}
$$

Suppose that $\cap\left\{V_{\mu_{(i)}}: i=1, \cdots, k\right\} \neq \varnothing$ and $x \in \cap\left\{V_{\mu(i)}: i=1, \cdots, k\right\}$. Then the set $s t(x, V)$ is contained in the open set $O y$ for some point $y \in Y$. Since $x \in \cap\left\{V_{\mu(i)}: i=1, \cdots, k\right\}$ then

$$
s t(x, \mathscr{W}) \cap F_{\mu(i)} \neq \varnothing \text { for } i=1, \cdots, k .
$$

Consequently, $O y \cap F_{\mu(i)} \neq \varnothing$ and by virtue of (12)

$$
y \in \cap\left\{F_{\mu(i)}: i=1, \cdots, k\right\} \text {. }
$$

This proves property (13). Let us take for every $\mu \in \mathscr{C}$ a set $W_{\mu}$ such that $\bar{W}_{\mu} \subset V_{\mu}$ and such that property (ii) holds. Then property (iii) follows from (13).

Lemma 2.5. Let $F=\left\{F_{\mu}: \mu \in \mathscr{C}\right\}$ be a locally finite collection

${ }^{8}$ By $\operatorname{st}(A, B)$, where $A \subset X$, and $B$ is a system of sets in $X$, we denote a star of a set $A$ with respect to a system $B$. 
of closed sets of order $r$ in a space $X$ and $\mathscr{W}=\left\{U_{\mu}: \mu \in \mathscr{C}\right\}$ be a collection of open sets such that $F_{\mu} \subset U_{\mu}$ for $\mu \in \mathscr{L}$. Then there exist closed sets $C_{i} i=1, \cdots, r$ such that

(i) Every set $C_{i} i=1, \cdots, r$ is a union of closed sets $\left\{C_{\nu}^{i}: \nu \in\right.$ $\mathscr{C}$ \} forming a discrete system in $X$, and every set $C_{\nu}^{i}$ is contained in some set $U_{\mu} \in \mathscr{\mathscr { C }}$.

(ii) $\bigcup_{i=1}^{r} C_{i}=\bigcup\left\{F_{\mu}: \mu \in \mathscr{C}\right\}$.

Proof. We shall prove the lemma by induction on $r$. Let $\Phi=$ $\cup\left\{F_{\mu}: \mu \in \mathscr{L}\right\}$. For $r=1$ the lemma is true because we can consider $C_{1}=\Phi$. Assume that the lemma has been proved for $(r-1)$ and $W=\left\{W_{\mu}: \mu \in \mathscr{K}\right\}$ be a collection of open sets, satisfying the hypothesis of Lemma 2.4. Obviously,

$$
\text { The system } W(\mathbf{1})=\left\{\bar{W}_{\mu}: \mu \in \mathscr{C}\right\} \text { has order } \leqq r .
$$

Moreover, the system $W(2)=\left\{F r W_{\mu}: \mu \in \mathscr{M}\right\}$ has an order $\leqq r-1$ on $\Phi$. Indeed, let $x \in \Phi \cap \cap\left\{F r W_{\mu_{(i)}}: i=1, \cdots, r\right\}$ then there exists such index $\mu_{(0)}$ that $x \in W_{\mu_{(0)}} \cap \Phi$. Therefore $x \in \cap\left\{\bar{W}_{\mu_{(i)}}: i=0, \cdots, r\right\}$ which contradicts the condition (14). By Lemma 2.4 we can construct a locally finite open collection of sets $Q=\left\{Q_{\mu}: \mu \in \mathscr{M}\right\}$ such that

$$
Q \text { has an order } \leqq r-1 \text { on } \Phi \text { and }
$$

$\operatorname{Fr} W_{\mu} \subset Q_{\mu} \subset U_{\mu}(\mu \in \mathscr{C})$. For any $\mu$ we consider an open set $P_{\mu}$ such that

$$
\operatorname{Fr} W_{\mu} \subset P_{\mu} \subset \bar{P}_{\mu} \subset Q_{\mu} \subset U_{\mu}
$$

Set

$$
\begin{gathered}
C_{1}=\Phi \backslash \cup\left\{P_{\mu}: \mu \in \mathscr{C}\right\} \\
D_{\nu}=\left(\bar{W}_{\nu} \cap \Phi\right) \backslash \cup\left\{P_{\mu}: \mu \in \mathscr{C}\right\} .
\end{gathered}
$$

Then from conditions (17), (18) it follows that

$$
C_{1}=\cup\left\{D_{\mu}: \mu \in \mathscr{C}\right\} \text {. }
$$

Assume that the set $\mathscr{C}$ is well ordered and put

$$
C_{\mu}^{\prime}=D_{\mu} \backslash \cup\left\{D_{\nu}: \nu<\mu\right\} \text {. }
$$

By condition (16) we have $\bar{W}_{\nu} \cup P_{\nu}=W_{\nu} \cup P_{\nu}$, then, by virtue of (18), (20)

$$
\begin{gathered}
C_{\mu}^{\prime}=D_{\mu} \backslash\left(\cup\left\{\bar{W}_{\nu}: \nu<\mu\right\} \cup\left\{P_{\mu}: \mu \in \mathscr{C}\right\}\right)=D_{\mu} \\
\mid \cup\left\{W_{\nu} \cup P_{\nu}: \nu<\mu\right\} \cup\left(P_{\mu}: \mu \in M\right\} .
\end{gathered}
$$


From conditions (20), (21) it follows that sets $C_{\mu}^{\prime}$ are closed and disjoint. Since $C_{\mu}^{\prime} \subset D_{\mu} \subset W_{\mu}$ the system $\left\{C_{\mu}^{\prime}: \mu \in \mathscr{C}\right\}$ is locally finite and, consequently, discrete. Since by virtue of (19), (20)

$$
\cup\left\{C_{\mu}^{\prime}: \mu \in \mathscr{C}\right\}=\bigcup\left\{D_{\mu}: \mu \in \mathscr{C}\right\}=C_{1}
$$

the set $C_{1}$ satisfies the condition (i) for $i=1$. From (15), (16) it follows that the system $P=\left\{\bar{P}_{\mu}: \mu \in \mathscr{C}\right\}$ has order $\leqq r-1$ on $\Phi$. Applying inductive assumption to locally finite closed system $P$ and open system $Q=\left\{Q_{\mu}: \mu \in \mathscr{C}\right\}$ we can find closed sets $C_{2}, \cdots, C_{r}$ satisfying conditions (i) (since $Q_{\mu} \subset U_{\mu}$ ) and the following condition:

$$
\cup\left\{C_{i}: i=2, \cdots, r\right\}=\bigcup\left\{\bar{P}_{\mu} \cap \Phi: \mu \in \mathscr{M}\right\} .
$$

By property (17) we obtain the equality $\cup\left\{C_{i}: i=1, \cdots, r\right\}=\Phi$.

CoROLlaRY 2.4. Let $U$ be an open covering of a space $X$, and ord $U \leqq r$. Then there exists a closed refinement of the covering $U$, consisting of $r$ discrete systems.

Proof. Using paracompactness of a space $X$ we can get closed locally finite combinatorial refinement $F$ of the covering $U$ with order $F \leqq r$. Then, our corollary follows from Lemma 2.5.

CoROLlaRY 2.5. For a space $X$ the following conditions are equivalent:

(a) $d_{k}(X)<\varepsilon$

(b) $X$ is a union of $k$ closed sets $C_{i}(1 \leqq i \leqq k)$ such that every set $C_{i}$ is a union of closed sets $\left\{C_{\mu}^{i}: \mu \in \mathscr{M}\right\}$ forming a discrete collection in $X$, and diameter $C_{\mu}^{i}<\varepsilon$ for each pair $i, \mu$.

(c) $X$ is a union of $k$ closed sets $C_{i}(1 \leqq i \leqq k)$ such that $d_{1}\left(C_{i}\right)<$ $\varepsilon$ for every $i \leqq k$.

(d) $X$ is a union of $k$ open sets $U_{i}(1 \leqq i \leqq k)$ such that $d_{1}\left(U_{i}\right)<$ $\varepsilon$ for each $i \leqq k$.

Proof. $(a) \Rightarrow(b)$. Let $d_{k}(X)<\varepsilon$, then there exists an open covering $U$ with mesh $U<\varepsilon$ and order $U \leqq k$. Then, using Corollary 2.4 we obtain assertion (b).

$(b) \Rightarrow(c)$. For proving it is sufficient to note that sets $C_{\mu}^{i}$ are open in $C_{i}$.

(c) $\Rightarrow(\mathrm{d})$. By virtue of (c) for each $i \leqq k$ there exists a covering $\mathscr{V}_{i}=\left\{C_{\mu}^{i}: \mu \in \mathscr{M}\right\}$ where sets $C_{\mu}^{i}$ are open in $C_{i}$ and disjoint, and have the diameter $<\varepsilon$. Therefore the collection $\mathscr{V}_{i}$ is discrete in $X$. Since $X$ is paracompact, we can find open sets $U_{\mu}^{i} \mu \in \mathscr{M}$ such that 


$$
C_{\mu}^{i} \subset U_{\mu}^{i}, \operatorname{diam} U_{\mu}^{i}<\varepsilon, U_{\mu}^{i} \cap U_{\mu^{\prime}}^{i}=\phi \text { for } \mu \neq \mu^{\prime} .
$$

Put $U_{i}=\bigcup\left\{U_{\mu}^{i}: \mu \in \mathscr{C}\right\}$. Then, the assertion (d) clearly holds.

Since a union of $k$ system of order $\leqq 1$ has an order $\leqq k$, we obtain the implication (d) $\Rightarrow(a)$.

\section{The Main Lemma.}

Lemma 3.1 (The Main Lemma). Let $S=\left\{S_{i}: i=1,2, \cdots\right\}$ be a countable locally finite system of closed sets in n-dimensional space $X$ and let $S$ be g.p, and $\mathscr{U}=\left\{U_{\mu}: \mu \in \mathscr{C}\right\}$ be an arbitrary open covering of $X$. Let also $\operatorname{dim} S_{i} \leqq n-1, i=1,2, \cdots, k \leqq n-1, n=$ $2,3,4, \cdots, D(n, k)=[n /(k+1)], \varepsilon>0$ and let $\left\{F_{r}, G_{r}\right\}(r=1, \cdots, D(n$, $k)$ ) be a system of pairs of disjoint closed sets in $X$ such that for any $i$ and for $r \leqq D(n, k)$

(1) either $S_{i} \cap F_{r}=\varnothing$ or $S_{i} \cap G_{r}=\varnothing$. Then there exist sets $D_{r} 1 \leqq r \leqq D(n, k)$ such that

(2) $D_{r}$ is a partition between $F_{r}$ and $G_{r}$.

(3) For the set $R=\cap\left\{D_{r}: 1 \leqq r \leqq D(n, k)\right\}$ we have $d_{k}\left(R \cap S_{i}\right)<$ $\varepsilon(i=1,2, \cdots)$.

(4) $\operatorname{dim} R \leqq \operatorname{dim} X-D(n, k)$.

(5) The set $R \cap S_{i}$ is a union of $k$ closed sets $L_{1}^{i}, \cdots, L_{k}^{i}$ such that every set $L_{j}^{i}$ is a union of closed sets forming a discrete system, and every element of this system is contained in some set $U_{\mu} \in \mathscr{Q}$.

Proof. By virtue of Corollary 2.3 we can find closed sets $C_{i}^{j} j, i=$ $1,2, \cdots$, such that for any pair $(i, j)$

(6) the system $S^{\prime}=S \cup\left\{C_{i}^{j}: i, j=1,2, \cdots\right\}$ is g.p.

(7) $\operatorname{dim} C_{i}^{j} \leqq n-1$.

(8) $d_{1}\left(X \backslash C_{i}^{j}\right)<\varepsilon$.

(9) The set $X \backslash C_{i}^{j}$ is a union of collection $B_{i}^{j}=\left\{U_{i \alpha}^{j}: \alpha \in A_{i j}\right\}$ of disjoint open sets and each $U_{i \alpha}^{j}$ is contained in some element $U_{\mu} \in \mathscr{U}$. Let us consider a system of closed sets

$$
\mathscr{P}=\left\{P_{i}: P_{i}=S_{i} \cap \cap\left\{C_{i}^{j}: j=1,2, \cdots, k\right\}\right\} .
$$

Since

$$
S_{i} \backslash P_{i}=\cup\left\{S_{i} \backslash C_{i}^{j}: j=1, \cdots, k\right\} \subset \cup\left\{X \backslash C_{i}^{j}: j=1, \cdots, k\right\}
$$

by virtue of (8) and Corollary 2.5

$$
d_{k}\left(S_{i} \backslash P_{i}\right)<\varepsilon .
$$

By virtue of (6), (7), (10) we have 


$$
\begin{aligned}
\operatorname{dim} \cap & \left\{P_{i(m)}: 0 \leqq m \leqq D(n, k)\right\} \\
= & \operatorname{dim} \cap\left\{S_{i(m)} \cap \cap\left\{C_{i(m)}^{j}: j=1, \cdots, k\right\}: 0 \leqq m \leqq D(n, k)\right\} \\
\leqq & \max \left\{\operatorname{dim} S_{i(m)}, \operatorname{dim} C_{i(m)}^{j}: 0 \leqq m \leqq D(n, k)\right\} \\
& -(D(n, k)+1)(k+1)+1 \\
= & (n-1)-(D(n, k)+1)(k+1)+1<n \\
& -([n /(k+1)]+1)(k+1)<0 .
\end{aligned}
$$

Consequently,

$$
\text { The order of } \mathscr{P} \leqq \mathscr{D}(n, k) \text {. }
$$

Since the system $S$ is locally finite and $P_{i} \subset S_{i}$ the system $\mathscr{P}$ is also locally finite. By virtue of (1) there exist such open sets $O_{i} \supset$ $P_{i}$ that

$$
\text { either } O_{i} \cap F_{r}=\varnothing \text { or } O_{i} \cap G_{r}=\varnothing 1 \leqq r \leqq D(n, k) .
$$

By Lemma 2.5 there exist $D(n, k)$ closed sets $C_{1}, \cdots, C_{D(n, k)}$ such that

$$
\cup\left\{C_{r}: r=1, \cdots, D(n, k)\right\}=P=\cup\left\{P_{i}: i=1,2, \cdots\right\} \text {. }
$$

(16) Every set $C_{r}$ is a union of a discrete collection of closed sets $\left\{C_{r}^{\mu}: \mu \in \mathscr{C}_{r}\right\}$ and each set $C_{r}^{\mu}$ is contained in some set $O_{i}$.

From (14) and (16) it follows that

$$
\text { Either } C_{r}^{\mu} \cap F_{r}=\varnothing \text { or } C_{r}^{\mu} \cap G_{r}=\varnothing \text { for } r \leqq D(n, k) \text {. }
$$

Put $F_{r}^{\prime}=F_{r} \cup\left\{C_{r}^{\mu}: C_{r}^{\mu} \cap F_{r} \neq \varnothing, \mu \in \mathscr{C}_{r}\right\}, G_{r}^{\prime}=G_{r} \cup\left\{C_{r}^{\mu}: C_{r}^{\mu} \cap F_{r}=\varnothing\right.$, $\mu \in \mathscr{M}_{r}$ \}. Obviously, $F_{r}^{\prime}$ and $G_{r}^{\prime}$ are disjoint closed sets and

$$
\begin{gathered}
F_{r}^{\prime} \cup G_{r}^{\prime} \supset C_{r} \\
F_{r}^{\prime} \supset F_{r}, \quad G_{r}^{\prime} \supset G_{r} .
\end{gathered}
$$

Therefore, we can find sets $D_{r} r \leqq D(n, k)$ such that $D_{r}$ is a partition between $F_{r}^{\prime}$ and $G_{r}^{\prime}$ and

$$
\operatorname{dim} \cap\left\{D_{r}: r=1, \cdots, D(n, k)\right\} \leqq \operatorname{dim} X-D(n, k) .
$$

Put

$$
R=\cap\left\{D_{r}: r=1, \cdots, D(n, k)\right\} .
$$

Then condition (4) follows from (21) and (20). The condition (2) follows from (19). From (18) it follows that $D_{r} \cap C_{r}=\varnothing$. Consequently, by virtue of (15), (21) 


$$
\begin{aligned}
& R \cap P= \cap\left\{D_{r}: r=1, \cdots, D(n, k)\right\} \\
& \cap\left(\cup\left\{C_{r}: r=1, \cdots, D(n, k)\right\}\right)=\varnothing .
\end{aligned}
$$

Hence

$$
R \cap S_{i} \subset S_{i} \backslash P \subset S_{i} \backslash P_{i}
$$

and by virtue of (12) the condition (3) is satisfied. By virtue of (9), (11), (22)

$$
R \cap S_{i} \subset \cup\left\{U_{i \alpha}^{j} \cap R \cap S_{i}: U_{i \alpha}^{j} \in B_{i^{\prime}}^{j} j=1, \cdots, k\right\}
$$

and collection $\mathscr{C}=\left\{U_{i \alpha}^{j} \cap R \cap S_{i}: U_{i \alpha}^{j} \in B_{i^{j}}^{j} j=1, \cdots, k\right\}$ has an order $\leqq k$ and any element of $\mathscr{V}$ is contained in some $U_{\mu} \in \mathscr{W}$. By virtue of Corollary 2.4 we now obtain property (5).

Lemma 3.2. Let $V \subset Z$ and $V=K \cup \cup\left\{R_{i}: i=1,2, \cdots\right\}$, where the sets $R_{i}$ are open-closed in $V, K$ is a closed set in $Z$ and

If

(i ) $R_{i} \cap R_{j}=R_{i} \cap K=\varnothing$ for $i \neq j$.

(ii) $\lim _{i \rightarrow \infty} d_{1}\left(R_{i}\right)=0$

then for any point $x \in K$ there is an arbitrary small neighborhood $O x$ in $Z$ such that

(iii) $\operatorname{FrOx} \subset Z \backslash \cup\left\{R_{i}: i=1,2, \cdots\right\}$.

Proof. Let $\varepsilon>0$. By virtue of (i) and (ii) there exist such disjoint open in $V$ sets $R_{i \alpha} i=1,2, \cdots, \alpha \in A_{i}$ so that $R_{i}=\cup\left\{R_{i \alpha}\right.$ : $\alpha \in A\}$, $\operatorname{diam} R_{i \alpha}<\varepsilon_{i}, \lim _{i \rightarrow \infty} \varepsilon_{i}=0$. Let $N$ be an integer such that for $i>N \varepsilon_{i}<\varepsilon / 4$. Then there exists a number $\delta(0<\delta<\varepsilon / 4)$ such that the neighborhood $O_{\delta}(x)^{10}$ doesn't intersect $R_{i}$ for $i<N$. Put

$$
O x=O_{\delta}(x) \cup \cup\left\{R_{i \alpha}: R_{i \alpha} \cap O_{\delta}(x) \neq \varnothing, i=1,2, \cdots, \alpha \in A_{i}\right\} .
$$

Obviously the diameter $O x \leqq \operatorname{diam} O_{\delta}+2 \sup \left\{\operatorname{diam} R_{i \alpha}: i=1,2, \cdots\right.$, $\left.\alpha \in A_{i}\right\}<\varepsilon / 2+\varepsilon / 2=\varepsilon$. Since sets $R_{i \alpha}$ are disjoint property (iii) follows from equality (24).

LEMMA 3.3. Let a space $Z$ have the representation:

(i ) $Z=K \cup \cup\left\{L_{i}^{j}: j=1, \cdots, k, i=1,2, \cdots\right\}$ where $K$ is a finite dimensional closed set and

(ii) Sets $L_{i}^{j}(i=1,2, \cdots)$ are open-closed in $K^{j}=K \cup \cup\left\{L_{i}^{j}: i=\right.$ $1,2, \cdots\}$, are open-closed in $K^{j}=K \cup \cup\left\{L_{i}^{j}: i=1,2, \cdots\right\}, K \cap L_{i}^{j}=$ $L_{i}^{j} \cap L_{i}^{j}=\varnothing$ for $i \neq i^{\prime}$.

(iii) $\lim _{i \rightarrow \infty} d_{1}\left(L_{i}^{j}\right)=0$.

Then, if

${ }^{10}$ For any set $\mathscr{M} \subset X$ by $O_{\delta}(\mathscr{M})$ we denote a $\delta$-neighborhood of the $\mathscr{M}$ in a space $X$. 
(iv) $\quad$ ind $(Z \backslash K) \leqq \alpha, \alpha \geqq \omega_{0}$

we have

(v) ind $Z \leqq \alpha+(k-1)$.

Proof. By virtue of (iv) it is sufficient to prove that

$$
\operatorname{ind}_{x} Z \leqq \alpha+(k-1) \text { if } x \in K \text {. }
$$

We shall prove this inequality by induction on $k$. Suppose that $k=1$, then by Lemma 3.2 there is an arbitrary small neighborhood of $x$ with boundary in $K$. Therefore, by virtue of finite dimensionality of $K$ we obtain (25). Suppose now $k>1$, and $\varepsilon>0$. Then by Lemma 3.2 for every $x \in K$ there exists a neighborhood $O x \ni x$ of the diameter $<\varepsilon$ such that $\operatorname{Fr} O x \subset Z \backslash \cup\left\{L_{i}^{k}: i=1,2, \cdots\right\}=Z_{1}$. (We consider that in Lemma $3.2 L_{i}^{k}=R_{i}$.) Since $Z_{1} \subset K \cup \cup\left\{L_{i}^{j}: j=\right.$ $1, \cdots, k-1, i=1,2, \cdots\}$, by inductive assumption we obtain ind $Z_{1} \leqq \alpha+(k-2)$. Consequently, ind $F r O x \leqq \alpha+(k-2)$. Hence, the inequality (25) and Lemma 3.3 are proved.

LemMA 3.4. Let a space $Z$ have the representation

$$
Z=K \cup \cup\left\{\Theta_{i}: i=1,2, \cdots\right\}
$$

where $K$ is a finite dimensional set, and sets $\Theta_{i}$ are open-closed in $Z$ and disjoint. If $\lim _{i \rightarrow \infty} d_{k}\left(\Theta_{i}\right)=0, K=Z \backslash \cup\left\{\Theta_{i}: i=1,2, \cdots\right\}$ then ind $Z \leqq \alpha+(k-1)$ where $\alpha \geqq \sup$ \{ind $\left.\Theta_{i}: i=1,2, \cdots\right\}$.

Proof. Let $d_{k}\left(\Theta_{i}\right)<\varepsilon_{i}$ and $\lim _{i \rightarrow \infty} \varepsilon_{i}=0$ then by Corollary 2.5 there exist closed sets $L_{i}^{j} j=1, \cdots, k$, such that $d_{1}\left(L_{i}^{j}\right)<\varepsilon_{i}, \cup\left\{L_{j}^{i}\right.$ : $j=1, \cdots, k\}=\Theta_{i}(i=1,2, \cdots)$. Therefore $Z=K \cup \cup\left\{L_{i}^{j}: i=1,2 \cdots\right.$, $j=1, \cdots, k\}$. Obviously, all conditions of Lemma 3.3 are satisfied. Consequently Lemma 3.4 follows from Lemma 3.3.

\section{Compacta $R_{\beta}$.}

Definition 4.1. Let $\left\{Y_{\mu}: \mu \in \mathscr{C}\right\}$ be a collection of spaces and $\{p\}$ be some point. Then by $\omega\left(p, Y_{\mu}: \mu \in \mathscr{C}\right)$ we denote a space

$$
\{p\} \cup \cup\left\{Y_{\mu}: \mu \in \mathscr{C}\right\}, Y_{\mu} \cap Y_{\mu^{\prime}}=Y_{\mu} \cap\{p\}=\varnothing \text { for } \mu \neq \mu^{\prime}
$$

where in set $U\left\{Y_{\mu}: \mu \in \mathscr{M}\right\}$ the topology is defined as in a discrete union of spaces $Y_{\mu}$ and in the point $\{p\}$ it is defined by the open basis:

$$
\omega\left(p, Y_{\mu}: \mu \in \mathscr{M}\right) \backslash \cup\left\{Y_{\mu(i)}: i=1,2, \cdots, k\right\}, \mu(i) \in \mathscr{M}, k=1,2, \cdots .
$$

We note, that if the set $\mathscr{M}$ is countable, and $Y_{\mu}$ are compact 
metric spaces, then $\omega\left(p, Y_{\mu}: \mu \in \mathscr{C}\right)$ is also a compact metric space.

LEMma 4.0. If the set $\mathscr{C}$ is countable, then for any metric on the set $\omega\left(Y_{\mu}: \mu \in \mathscr{C}\right)$ and any $\varepsilon>0$ the inequality diam $Y_{\mu}<\varepsilon$ holds for all but only finitely many $\mu \in \mathscr{K}$.

The lemma is evident.

Definition 4.2. (See [14] Luxemburg). Let $\left(A_{n+1}, B_{n+1}\right), \cdots$, $\left(A_{n+k}, B_{n+k}\right)$ be a fixed collection of pairs of opposite faces in Euclidean cube $I^{n+k}$. If $C_{n+i}$ is a partition between $A_{n+i}$ and $B_{n+i} i=$ $1, \cdots, k$ and

$$
\operatorname{dim} \cap\left\{C_{n+i}: i=1, \cdots, k\right\}=n
$$

then the set $Q=\cap\left\{C_{n+i}: i=1, \cdots, k\right\}$ is called $n$-dimensional pseudocube. (We note that $Q$ is always of dimension $\geqq n$, Hurewicz ([4], p. 40).) The rest $n$ pairs $A_{i}, B_{i}$ of opposite faces in cube $I^{n+k}$ are called improper faces of pseudocube. The intersections $F_{i}=$ $A_{i} \cap Q, G_{i}=B_{i} \cap Q(1 \leqq i \leqq n)$ are called opposite faces of the pseudocube $Q$.

Lemma 4.1. The product of $n$-dimensional and k-dimensional pseudocubes is $(n+k)$ dimensional pseudocube.

The lemma is evident.

Lemma 4.2. Let $Q^{n}$ be an n-dimensional pseudocube and ( $F_{i}$, $\left.G_{i}\right) i=1, \cdots, l, l \leqq n$ are $l$-pairs of its opposite faces. Then for any collection of partitions $C_{i}$ between these pairs

(i) $\operatorname{dim} \cap\left\{C_{i}: i=1, \cdots, l\right\} \geqq n-l$.

(ii) If $\operatorname{dim} \cap\left\{C_{i}: i=1, \cdots, l\right\} \leqq n-l$ then the set $R=\cap\left\{C_{i}\right.$ : $i=1, \cdots, l\}$ is an $(n-l)$ dimensional pseudocube.

Proof. Let the pseudocube $Q^{n}$ has a representation

$$
Q^{n}=\cap\left\{D_{n+j}: j=1, \cdots, k\right\}
$$

where sets $D_{n+j}$ are partitions between opposite spaces of some cube $I^{n+k}$. Let

$$
A_{i} \cap Q^{n}=F_{i}, B_{i} \cap Q^{n}=G_{i}, i=1, \cdots, n
$$

where $A_{i}, B_{i}$ are proper faces of the pseudocube $Q^{n}$. Let $D_{i}$ be a partition between $A_{i}$ and $B_{i}$ in the cube $I^{n+k}$ such that $D_{i} \cap Q^{n}=C_{i}$. Then 


$$
\begin{gathered}
\cap\left\{C_{i}: i=1, \cdots, l\right\}=\cap\left\{D_{j+n}: j=1, \cdots, k\right\} \\
\cap \cap\left\{D_{i}: i=1, \cdots, l\right\}=R .
\end{gathered}
$$

The set $R$ is an intersection of $(k+l)$ partitions between opposite faces in cube $I^{n+k}$. Consequently $\operatorname{dim} R \geqq n+k-(k+l)=n-l$ (see Hurewicz [4] p. 40) and if also $\operatorname{dim} R \leqq n-l$ then $R$ is a pseudocube.

In this and in the next section by $\alpha$ we mean a limit ordinal number or 0 , and $n=0,1,2, \cdots$.

DEFINITION 4.3. We shall define for every ordinal number $\beta<\omega$, a class $\rho_{\beta}$ consisting of compacta. Suppose that $\beta$ is a finite number, then $\rho_{\beta}$ consists of all $\beta$-dimensional pseudocubes. If $\beta$ is a limit number, then $\rho_{\beta}$ consists of all compacta $R_{\beta}$ having the following representation

$$
R_{\beta}=\omega\left(p_{\beta} ; R_{\gamma}: \gamma \in \Gamma_{\beta}\right)
$$

where $p_{\beta}$ is an extra point, $R_{\gamma} \in \rho_{\gamma}$ and $\Gamma_{\beta}$ some cofinal subset in $\dot{\phi}_{\beta}=\{\gamma: \gamma<\beta\}$. If $\beta=J(\beta)+K(\beta), K(\beta)>0$, then class $\rho_{\beta}$ consists of all compacta $R_{\beta}$ such that

$$
R_{\beta}=R_{J(\beta)} \times R_{K(\beta)}, R_{J(\beta)} \in \rho_{J(\beta)}, R_{K(\beta)} \in \rho_{K(\beta)} .
$$

In what follows $R_{\beta}$ will denote an element of class $\rho_{\beta}$. Let us introduce some notations. In this and following sections we consider $\alpha$ to be a limit number or 0 and $n=0,1,2, \cdots$. If $\beta=\xi+1$ then put $\beta-1=\xi$. For every compactum $R_{\alpha+n}$ we have by definition: $R_{\alpha+n}=R_{\alpha} \times R_{n}$ for some $R_{\alpha} \in \rho_{\alpha^{\prime}} R_{n} \in \rho_{n}$. Obviously, $R_{\alpha+n}$ has the representation:

$$
R_{\alpha+n}=\bar{R}_{n} \cup \cup\left\{R_{\gamma} \times R_{n}: \gamma \in \Gamma_{\alpha}\right\}
$$

where $\Gamma_{\alpha}$ is some cofinal subset in $\phi_{\alpha}=\{\gamma: \gamma<\alpha\}$, and $\bar{R}_{n}=\left\{p_{\alpha}\right\} \times$ $R_{n}$ and $p_{\alpha}$ is an extra point in $R_{\alpha}$. Representation (1) we shall call a standard representation of a compactum $R_{\alpha+n}$.

Definition 4.4. (See Smirnov [17].) For any $\beta<\omega_{1}$, we define a class of compacta $\Pi_{\beta}$. If $\beta<\omega_{0}$, then $\Pi_{\beta}$ consists of all $\beta$ dimensional compacta. If $\beta$ is a limit number, then $\Pi_{\beta}$ consists of all compacta $X$ such that $X=\omega\left(p ; Y_{r}: Y_{r} \in \Pi_{r}: \gamma<\beta\right)$ where $p$ is an extra point. Moreover, if $\beta=J(\beta)+K(\beta), \quad X \in \Pi_{J(\beta)}$, $Y \in \Pi_{K(\beta)}$ then $X \times Y \in \Pi_{\beta}$. Thus, for any $\beta<\omega_{1}$, we have defined the class $\Pi_{\beta}$. 
In [17], (Smirnov) it was proved, that for any $\beta<\omega_{0}$

$$
\text { Ind } X \leqq \beta \text { if } X \in \Pi_{\beta} \text {. }
$$

LEMMA 4.3. Every compactum $R_{\beta}$ is contained in some compactum $X \in \Pi_{\beta}$.

Proof. Let $Y \in \Pi_{\beta}$, then from Definitions 4.3 and 4.4 it follows that a discrete sum $X=Y \oplus R_{\beta}$ is an element of $\Pi_{\beta}$.

CoROLLARY 4.1. ind $R_{\beta} \leqq$ Ind $R_{\beta} \leqq \beta$.

Proof. It is directly derived from (2) and Lemma 4.3.

LEMmA 4.4. For any $q$-dimensional compactum $K$ the following inequality holds:

$$
\text { Ind } R_{\beta} \times K \leqq \text { Ind } R_{\beta}+q \leqq \beta+q \text {. }
$$

Proof. Let $\beta=\alpha+n$, then $R_{\beta}=R_{\alpha+n}=R_{\alpha} \times R_{n}, R_{\beta} \times K=$ $R_{\alpha} \times\left(R_{n} \times K\right)$. Let $X \supset R_{\alpha}$ and $X \in \Pi_{\alpha}$ then by Definition $4.4 X \times$ $\left(R_{n} \times K\right) \in \prod_{\alpha+s} s=\operatorname{dim} R_{n} \times K \leqq n+q$. From property (2) it follows that Ind $\left(X \times R_{n} \times K\right) \leqq \alpha+s \leqq \alpha+n+q=\beta+q$. Since, obviously $R_{\beta} \times K \subset X \times R_{n} \times K$, Ind $R_{\beta} \times K \leqq \beta+q$.

Let (1) be a standard representation of a compactum $R_{\alpha+n}$, then by $i$ we denote the natural homeomorphism $i: \mathbb{R}_{n} \rightarrow R_{n}$.

LEMMA 4.5. Let (1) be a standard representation of a compactum $R_{\alpha+n}$ and $F$ be a closed subset in $R_{\alpha+n}$ such that $F \subseteq \bar{R}_{n} \cup \cup$ $\left\{R_{\gamma} \times C_{r}: \gamma \in \Gamma_{\alpha}\right\}$

$$
\text { where } C_{r} \subset R_{n} \operatorname{dim} C_{r} \leqq k, k=-1,0,1, \cdots .
$$

Then for any pair of closed disjoint sets $\left(A, A^{\prime}\right), A \cup A^{\prime} \subset \bar{R}_{n}$ there exists a partition $D$ in compactum $R_{\alpha+n}$ between $A$ and $A^{\prime}$ such that

$$
D \cap R_{r} \times C_{r} \subset R_{r} \times C_{\gamma}^{\prime}
$$

where $C_{r}^{\prime} \subset R_{n^{\prime}} \operatorname{dim} C_{r}^{\prime} \leqq k-1$ for $k \geqq 1$ and $D \cap R_{r} \times C_{r}=\varnothing$ for $k \leqq 0$.

Proof. Let $D_{0}$ be an arbitrary partition in $R_{n}$ between $i(A)$ and $i\left(A^{\prime}\right)$, then there exist open in $R_{n}$ sets $U$ and $V$ such that

$$
R_{n} \backslash D_{0}=U \cup V, A \subset U, A^{\prime} \subset V .
$$


Consequently, there exists a sequence of pairs of open sets $U_{n}, V_{n}$ such that

$$
\begin{gathered}
A \subset \bar{U}_{n} \subset U_{n+1}, A^{\prime} \subset \bar{V}_{n} \subset V_{n+1}, \cup\left\{U_{n}: n=1,2, \cdots\right\}=U, \\
\cup\left\{V_{n}: n=1,2, \cdots\right\}=V .
\end{gathered}
$$

Let $\tau: N \rightarrow \Gamma_{\alpha}$ be a bijective mapping of the set of all integers $N$ onto $\Gamma_{\alpha}$. Then there exists a partition $D_{n}$ in $R_{n}$ between $i\left(\bar{U}_{n}\right)$ and $i\left(\bar{V}_{n}\right)$ such that:

$$
\begin{gathered}
\operatorname{dim}\left(D_{n} \cap C_{\tau(n)}\right) \leqq k-1 \text { for } k \geqq 1 \text { and } \operatorname{dim} D_{n} \cap C_{\tau(n)}=-1 \\
\text { for } k \leqq 0
\end{gathered}
$$

We put

$$
D=D_{0} \cup \cup\left\{R_{\tau(n)} \times D_{n}: n=1,2, \cdots\right\} .
$$

Then by virtue of (7)

$$
D \cap R_{r} \times C_{r}=R_{r} \times\left(D_{\tau^{-1}(\gamma)} \cap C_{r}\right)=R_{\gamma} \times C_{\gamma}^{\prime}
$$

where $C_{\gamma}^{\prime}=D_{\tau^{-1}(r)} \cap C_{r}$ and $\operatorname{dim} C_{r}^{\prime} \leqq k-1$ for $k \geqq 1$ and $C_{\gamma}^{\prime}=\varnothing$ for $k \leqq 0$. By virtue of (5), (6), (8) $D$ is a partition between $A$ and $A^{\prime}$ in $R_{\alpha+n}$.

LEMMA 4.6. If conditions of the Lemma 4.5 are satisfied, then for the set $F$ Ind $F \leqq \alpha+k$ for $k \geqq 0$ and Ind $F \leqq n$ for $k<0$.

Proof. We shall prove the lemma by induction on $k$. Suppose $k=-1$ or $\alpha=0$ then the assertion is evident. Let $k \geqq 0, \alpha \geqq \omega_{0}$ and $\left(A, A^{\prime}\right)$ be an arbitrary pair of disjoint closed sets in $F$. By virtue of Lemma 4.5 there exists a partition $D$ between $A \cap \bar{R}_{n}$ and $A^{\prime} \cap \bar{R}_{n}$ such that property (4) holds. Consequently, $F \cap D \subset$ $\bar{R}_{n} \cup \cup\left\{R_{\gamma} \times C_{r}^{\prime}: \gamma \in \Gamma_{\alpha}\right\}, \operatorname{dim} C_{r}^{\prime} \leqq k-1$ for $k \geqq 1$ and $D \cap F \subset \bar{R}_{n}$ for $k=0$. Therefore, by inductive assumption

$$
\text { Ind } D \cap F \leqq \alpha+k-1 \text {, or Ind } D \cap F \leqq n \text {. }
$$

Since $F$ is a compactum, there exists a finite collection of ordinal numbers $\gamma(1), \cdots, \gamma(s), s=1,2, \cdots$ such that for the set $X=F \backslash \cup$ $\left\{R_{\gamma(i)} \times C_{\gamma(i)}: i=1, \cdots, s\right\}$ we have

(10) The set $D \cap X$ is a partition in $X$ between $A \cap X$ and $A^{\prime} \cap X$. Let $Y=F \backslash X=\cup R_{\gamma(i)} \times C_{\gamma(i)}$, then from condition (3) and Lemma 4.4 it follows that

$$
\text { Ind } \begin{aligned}
Y & \leqq \max \left\{\text { Ind } R_{\gamma(i)} \times C_{\gamma_{(i)}}: i=1, \cdots, s\right\} \\
& \leqq \max \{\gamma(i)+k: i=1, \cdots, s\}<\alpha .
\end{aligned}
$$


Therefore, an arbitrary partition $D^{\prime}$ in $Y$ between $Y \cap A$ and $Y \cap A^{\prime}$ has the dimension Ind $D^{\prime}<\alpha$. Since $X \cap Y=\varnothing, X \cup Y=F$, then $(F \cap D) \cup D^{\prime}$ is a partition in $F$ between $A$ and $A^{\prime}$, and

$$
\begin{aligned}
& \text { Ind }(D \cap F) \cup D^{\prime} \leqq \max \left(\operatorname{Ind}(D \cap F), \text { Ind } D^{\prime}\right) \leqq \alpha+k-1 \\
& \quad \text { for } k \geqq 1, \\
& \text { Ind }(D \cap F) \cup D^{\prime}<\alpha \quad \text { for } k=0 .
\end{aligned}
$$

In any case Ind $(D \cap F) \cup D^{\prime}<\alpha+k$.

Lemma 4.7. Let $\left(F_{i}, G_{i}\right) i=1, \cdots, n$ be a fixed system of pairs of closed sets in a space $X, F_{i} \cap G_{i}=\varnothing$. If for any partition $C_{i}$ between these pairs we have

$$
\text { Ind } \cap\left\{C_{i}: i=1, \cdots, n\right\} \geqq \beta
$$

then for any $k \leqq n$

$$
\text { Ind } \bigcap_{i=1}^{k} C_{i} \geqq \beta+n-k
$$

and

$$
\text { Ind } X \geqq \beta+n \text {. }
$$

Proof. Let inequality (12) be false. Then there exist partitions $C_{k+1}, \cdots, C_{n}$ such that $C_{l}$ separates $F_{l}$ and $G_{l} k+1 \leqq l \leqq n$ and Ind $\cap$ $\left\{C_{i}: i=1, \cdots, k\right\}<\beta$. This contradicts inequality (11). From property (12) it follows that

Ind $C_{1} \geqq \beta+(n-1)$ for every partition between $F_{1}$ and $G_{1}$. Hence, inequality (13) holds.

Lemma 4.8. Let $R_{n}$ be a pseudocube, and $\left(A_{i}, B_{i}\right)(i=1, \cdots, k)$ $k \leqq n$ be any system of its opposite faces. Consider a system of pairs of closed subsets $\left(A_{i} \times R_{\beta}, B_{i} \times R_{\beta}\right)$ in the compactum $R_{\beta+n} \in \rho_{\beta+n}$, $R_{\beta+n}=R_{\beta} \times R_{n} \beta<\omega_{1}$. Then for any collection of partitions between these pairs we have:

$$
\text { Ind } \cap\left\{C_{i}: i=1, \cdots, k\right\} \geqq \beta+n-k .
$$

We shall prove the lemma by induction on $\beta$. For $\beta<\omega_{0}$ our assertion follows from Lemmas 4.1, 4.2. Suppose $\beta=\alpha+q \geqq \omega_{0}$, $q=0,1, \cdots$ and for all $\gamma<\beta$ our lemma is proved. We can suppose $R_{\beta}=R_{\alpha} \times R_{q}$. Let $A$ be a system of all opposite faces of pseudocube $R_{n+q}=R_{n} \times R_{q}$. Then the following system $\mathscr{B}=\left\{A_{i} \times R_{q}, B_{i} \times\right.$ $\left.R_{q}: i=1, \cdots, k\right\}$ is a subsystem of $A$. By virtue of Lemma 4.7 it is sufficient to prove that for any collection $\left\{D_{i}: i=1, \cdots, n+q\right\}$ of 
partitions in $R_{\beta+n}$ between distinct pairs of the system $\left(X \times R_{\alpha}\right.$, $\left.Y \times R_{\alpha}\right),(X, Y) \in A$ we have

$$
\text { Ind } \cap\left\{D_{i}: i=1, \cdots, n+q\right\} \geqq \alpha .
$$

Let $\bar{R}_{n+q} \cup \cup\left\{R_{\gamma} \times R_{n+q}: \gamma \in \Gamma_{\alpha}\right\}$ be a standard representation of a compactum $R_{\beta+n}=R_{\alpha+n+q}$. Let $\gamma \in \Gamma_{\alpha}$ then

$R_{\tau} \times R_{n+q}=R_{\gamma+n+q} \subset R_{\beta+n}, \quad \cap\left\{D_{i}: i=1, \cdots, n+q\right\} \cap R_{\alpha+n+q} \subset \cap$ $\left\{D_{i}: i=1, \cdots, n+q\right\}$. Since for every $i \leqq n+q$ the set $D_{i} \cap R_{\tau+n+q}$ is a partition between a pair $\left(X \times R_{\tau}, Y \times R_{r}\right),(X, Y) \in A$ by inductive assumption we obtain Ind $\cap\left\{D_{i} \cap R_{\gamma+n+q}: i=1, \cdots, n+q\right\} \geqq$ $\gamma$. Since $\sup \left\{\gamma: \gamma \in \Gamma_{\alpha}\right\}=\alpha$ we obtain inequality (14).

The following lemma is evident.

LEMMA 4.9. Let $Y=\omega\left(p ; Y_{i}: i=1,2, \cdots\right)$ then

$$
\begin{aligned}
& \text { Ind } Y=\sup \left\{\text { Ind } Y_{i}: i=1,2, \cdots\right\} \\
& \text { ind } \left.Y=\sup \text { (ind } Y_{i}: i=1,2 \cdots\right\} .
\end{aligned}
$$

Corollary 4.2. For any $\beta<\omega_{1}$ we have Ind $R_{\beta}=\beta$.

We shall prove the corollary by induction of $\beta$. Suppose $\beta$ is nonlimit ordinal number, then $R_{\beta}=R_{\alpha} \times R_{n}(n=1,2, \cdots)$. Let $(A$, $B$ ) be a pair of opposite faces of pseudocube $R_{n}$. Then by Lemma 4.8 for any partition $C$ between $A \times R_{\alpha}$ and $B \times R_{\alpha}$ in $R_{\beta}$ Ind $C \geqq$ $\alpha+n-1$. Consequently, Ind $R_{\beta} \geqq \beta$. The inequality Ind $R_{\beta} \leqq \beta$ follows from Corollary 4.1. Let $\beta$ be a limit number, then by definition $R_{\beta}=\omega\left(p_{\beta}: \beta \in \Gamma_{\beta}\right)$ and our assertion follows from the inductive assumption and Lemma 4.9.

Lemma 4.10. Every compactum $R_{\beta} \in \rho_{\beta}$ is weakly countable dimensional.

We shall prove this lemma by induction on $\beta$. If $\beta<\omega_{0}$, then our lemma is evident. Suppose all compacta $R_{\gamma}$ are countable dimensional for $\gamma<\beta$. If $\beta$ is the limit number, then $R_{\beta}=\omega\left(p_{\beta}\right.$ : $\left.R_{r}: \gamma \in \Gamma_{\beta}\right)$. Since $\beta<\omega_{1}, \Gamma_{\beta}$ is a countable set. Consequently, $R_{\beta}$ is a union of countable number of its weakly-countable dimensional closed subsets. Therefore, $R_{\beta}$ is also countable dimensional. If $\beta=$ $\alpha+n$, then $R_{\beta}=R_{\alpha} \times R_{n}$ and $R_{\alpha}$ is weakly countable dimensional by inductive assumption. Consequently, $R_{\beta}$ is also weakly countable dimensional.

5. The proof of Theorem 1.2. First we introduce some 
notations. For $k=0,1,2, \cdots$, put $\bar{m}(k)=k-[k / 2]$. Let $R_{n}$ be a pseudocube. Let us number all pairs of its opposite faces. Then by $A\left(s, t, R_{n}\right)$ we denote a subsystem of a system of all pairs of opposite faces such that $A\left(s, t, R_{n}\right)$ contains all pairs with numbers $s, \cdots, t(s \leqq t)$. By definition the system $A\left(s, t, R_{n}\right) \times R_{\alpha}$ consists of all pairs $\left(F \times R_{\alpha}, G \times R_{\alpha}\right)$ where $(F, G) \in A\left(s, t, R_{n}\right)^{11}$. Let $A$ be a system of pairs of sets, then for the set $X, A \wedge X$ denotes the system of pairs $(F \cap X, G \cap X)$ where $(F, G) \in A$. In this section we consider $\Gamma_{\alpha}=\{\gamma: \gamma<\alpha\}$.

Proposition 5.1. Let $R_{\alpha+n}=R_{\alpha} \times R_{n}, n \geqq 2$. Then for any system $A\left(1, m, R_{n}\right), m=\bar{m}(n)$, there exists a compactum $L_{\alpha+m} \subset R_{\alpha+n}$ such that

(i) Ind $L_{\alpha+m} \leqq \alpha+m$.

(ii) For any collection of partitions $D_{i}(i=1, \cdots, m)$ between the pairs of the system $A\left(1, m, R_{n}\right) \times R_{\alpha}$ we have:

(iii) ind $L_{\alpha+m}=\psi(\alpha+m)$.

$$
\text { Ind } \cap\left\{D_{i}: i=1, \cdots, m\right\} \cap L_{\alpha+m} \geqq \alpha
$$

We note, that from Lemma 4.7 and conditions (i), (ii) it follows that Ind $L_{\alpha+m}=\alpha+m$. We shall prove the proposition by induction on $\alpha$. Suppose $\alpha=0$ and $D_{m+1}, \cdots, D_{n}$ is a collection of partitions between the pairs of the system $A\left(m+1, n, R_{n}\right)$ such that for the set $L_{m}=\cap\left\{D_{m+i}: i=1, \cdots, n-m\right\}$ we have $\operatorname{dim} L_{m}=m$. Then by Lemma $4.2 L_{m}$ is $m$-dimensional pseudocube and $A\left(1, m, R_{n}\right) \cap L_{m}$ is a system of pairs of its opposite faces. Thus property (ii) follows from Lemma 4.2, and properties (i), (iii) are evident.

Suppose $\alpha=\alpha_{0} \geqq \omega_{0}$ and for any $\alpha<\alpha_{0}$ our proposition holds. Let $\widetilde{\Gamma}_{\alpha} \subset \Gamma_{\alpha}$ be a subset of $\Gamma_{\alpha}$ consisting of all ordinal numbers $\gamma$ with

(1) $K(\gamma)>m$.

Let $f: N \rightarrow \widetilde{\Gamma}_{\alpha}$ be a bijection of the set of all integers $n>0$ onto $\widetilde{\Gamma}_{\alpha}$. Further, since $\sigma$-locally finite open base in a compact space is obviously countable, by virtue of Corollary 2.2 we obtain an open basis $\mathscr{V}=\left\{V_{k}: k=1,2, \cdots\right\}$ in compactum $R_{n}$ such that

(2) $\operatorname{dim} \operatorname{Fr} V_{k} \leqq n-1$.

(3) the system $\left\{F r V_{k}: k=1,2, \cdots\right\}$ is g.p.

Obviously we can also require the following condition: either $V_{k} \cap$ $F_{i}=\varnothing$ or $V_{k} \cap G_{i}=\varnothing$ for $\left(F_{i}, G_{i}\right) \in A\left(1, n, R_{n}\right),(k=1,2, \cdots)$. If we apply Lemma 3.1 to the system $S_{p}=\left\{V_{k}: k \leqq p\right\}, k=1, \varepsilon=1 / p$, we obtain for any $p \in N$ a closed set $C_{p} \subset R_{n}$ such that

(4) $C_{p}$ is an intersection of [n/2] partitions between pairs of

${ }^{11}$ For $\alpha=0$ we consider that $A\left(1, m, R_{n}\right) \times R_{\alpha}=A\left(1, m, R_{n}\right)$. 
the system $A\left(m+1, n, R_{n}\right)$ in $R_{n}$.

(5) $\quad d_{1}\left(C_{p} \cap F r V_{k}\right)<1 / p(1 \leqq k \leqq p)$.

(6) $\operatorname{dim} C_{p} \leqq m=n-[n / 2]$.

From conditions (4), (6) and Lemma 4.2 it follows that $C_{p}$ is an $m$ dimensional pseudocube. Let $R_{\alpha+n}=\bar{R}_{n} \cup \cup\left\{R_{\gamma} \times R_{n}: \gamma \in \Gamma_{\alpha}\right\}$ be a standard representation of compactum $R_{\alpha+n}$. Put

(7) $\quad S_{\alpha+n}=\bar{R}_{n} \cup \cup\left\{R_{f(p)} \times C_{p}: p=1,2, \cdots\right\} \subset R_{\alpha+n}$.

We set $J(f(p))=\xi_{p}, K(f(p))=s(p), m+s(p)=n(p)$. Then by virtue of $(1)$

(8) $m \leqq[n(p) / 2], m \leqq n(p)-[n(p) / 2]=\bar{m}(n(p))$ and $R_{f(p)} \times C_{p} \in \rho_{f(p)+m}=\rho_{\xi p+n(p)}$. Let $R_{f}(p)=R_{\xi p} \times R_{s(p)}$. By virtue of Lemma 4.1 the set $R_{s(p)} \times C_{p}$ is $n(p)$-dimensional pseudocube. If $A\left(1, s(p), R_{s(p)}\right)=\left\{A_{j}, B_{j}: j \leqq s(p), A\left(1, m, C_{p}\right)=\left\{F_{i}, G_{i}: i \leqq m\right\}\right.$, then obviously

$$
\begin{aligned}
& A\left(1, n(p), R_{s(p)} \times C_{p}\right)=\left\{A_{j} \times C_{p}, B_{j} \times C_{p}, R_{s(p)}\right. \\
& \left.\quad \times F_{i}, R_{s(p)} \times G_{i}: i \leqq m, j \leqq s(p)\right\} .
\end{aligned}
$$

Let us number elements of the system $A\left(1, n(p), R_{s(p)} \times C_{p}\right)$ by such a way that a pair $R_{s(p)} \times F_{i}, R_{s(p)} \times G_{i}$ gets a number $i(i \leqq m)$. Let us apply inductive assumption to the system of pairs $A(1, m(p)$, $R_{s(p)} \times C_{p}$ ) and to the compactum $R_{f(p)} \times C_{p} \in \rho_{\xi_{p}+n(p)}$ where $m(p)=$ $n(p)-[n(p) / 2]=\bar{m}(n(p))$. Then there exists a compactum $L_{\xi_{p}+m(p)} \subset$ $R_{f(p)} \times C_{p} \subset R_{\alpha+n}$ such that:

( $\left.i_{p}\right)$ Ind $L_{\xi_{p}+m(p)} \leqq \xi_{p}+m(p)$.

( $\left.\mathrm{ii}_{\mathrm{p}}\right)$ For any collection of partitions $D_{i}^{p}(i=1, \cdots, m(p))$ in a compactum $R_{\xi_{p}+n(p)}=R_{f(p)} \times C_{p}=R_{\xi_{p}} \times\left(R_{s(p)} \times C_{p}\right)$ between pairs of the system $A\left(1, m(p), R_{s(p)} \times C_{p}\right) \times R_{\xi_{p}}$ we have:

$$
\text { Ind }\left(L_{\xi_{p}+m(p)} \cap \cap\left\{D_{i}^{p}: i=1, \cdots, m(p)\right\}\right) \geqq \xi_{p} .
$$

$\left(\mathrm{iii}_{\mathrm{p}}\right) \quad$ ind $L_{\xi_{p}+m(p)}=\psi\left(\xi_{p}+m(p)\right)$ and $L_{\xi_{p}+m(p)} \subset R_{f(p)} \times C_{p}=R_{\xi_{p}+n(p)} \subset R_{\alpha+n}$. We put

$$
L_{\alpha+m}=\bar{R}^{n} \cup \cup\left\{L_{\xi_{p}+m(p)}: p=1,2, \cdots\right\} \subset S_{\alpha+n} \subset R_{\alpha+n} .
$$

By virtue of (6), (7) and Lemma 4.6 Ind $L_{\alpha+m} \leqq$ Ind $S_{\alpha+m} \leqq \alpha+m$. Hence, the condition (i) holds. Let us prove property (ii). Let $D_{i}(i \leqq m)$ be a collection of partitions between the pairs of a system $A\left(1, m, R_{n}\right) \times R_{\alpha}$ and $p$ be an arbitrary integer $>0$. By virtue of chosen numeration of elements of the system $A\left(1, m, R_{s(p)} \times C_{p}\right)$ we have

$$
A\left(1, m, R_{s(p)} \times C_{p}\right) \times R_{\xi_{p}}=\left(A\left(1, m, R_{n}\right) \times R_{\alpha}\right) \wedge\left(R_{f(p)} \times C_{p}\right) .
$$

Put 


$$
D_{i}^{p}=D_{i} \cap R_{f(p)} \times C_{p} \subset L_{\alpha+m} .
$$

Then $D_{i}^{p}$ is a partition in compactum $R_{f(p)} \times C_{p}$ between a pair $A\left(i, i, R_{s(p)} \times C_{p}\right) \times R_{\xi_{p}}$. By virtue of $(8) m \leqq m(p)=n(p)-[n(p) / 2]$. Therefore, by Lemma 4.7 and condition ( $\mathrm{ii}_{\mathrm{p}}$ )

$$
\text { Ind }\left(\cap\left\{D_{i}^{p}: i=1, \cdots, m\right\} \cap L_{\xi_{p}+m(p)}\right) \geqq \xi_{p}+(m(p)-m) .
$$

Since $\xi_{p}+(m(p)-m)=J(\gamma)+(K(\gamma)+m)-[(K(\gamma)+m) / 2]-m=$ $J(\gamma)+K(\gamma)+[(K(\gamma)+m) / 2]$ for $\gamma=f(p)$ we have $\sup \left\{\xi_{p}+(m(p)-\right.$ $m): p \in N\}=\sup \{J(\gamma)+K(\gamma)+[(K(\gamma)+m) / 2]: \gamma<\alpha, K(\gamma)>m\}=\alpha$. Therefore by virtue of conditions (11), (12), property (ii) holds. We have noted above that from properties (i), (ii) follows the equality Ind $L_{\alpha+m}=\alpha+m$. Then, by virtue of Corollary 1.1

$$
\text { ind } L_{\alpha+m} \geqq \psi(\alpha+m) \text {. }
$$

Therefore, we have only to prove inequality:

$$
\operatorname{ind}_{x} L_{\alpha+m} \leqq \psi(\alpha+m)
$$

for any $x \in L_{\alpha+m}$. If $x \in L_{\xi_{p}+m(p)} \subset L_{\alpha+m}$ then inequality (14) follows from the inductive assumption. Indeed, by virtue of Lemma 1.1 (j) $\psi\left(\xi_{p}+m(p)\right) \leqq \psi(\alpha+m)$ and since $L_{\xi_{p}+m(p)}$ is obviously open in $L_{\alpha+m}$, inequality (14) follows from (iii ${ }_{\mathrm{p}}$ ). Therefore, by virtue of (9) it is sufficient to prove inequality (14) for $x \in \bar{R}_{n}$. Let us consider an open in $L_{\alpha+m}$ set $U=U(k, q)$, where

$$
U=V_{k} \times\left(R_{\alpha} \backslash \cup\left\{R_{f(p)}: p=1, \cdots, q\right\}\right) \cap L_{\alpha+m}, V_{k} \in \mathscr{C} .
$$

Since the system of open sets $\{U(k, q): k, 1=1,2, \cdots\}$ forms a basis in any point $x \in R_{n}$ it is sufficient to prove that

$$
\text { ind }\left(\operatorname{Fr} U \cap L_{\alpha+m}\right)<\psi(\alpha+m) \text {. }
$$

By virtue of (9), (7) and (15) we have

$$
\begin{aligned}
& F r U \cap L_{\alpha+m} \subset\left(F r V_{k} \times R_{\alpha}\right) \cap L_{\alpha+m} \subset \bar{R}_{n} \cup \\
& \quad \cup\left\{\left(F r V_{k} \times R_{\alpha} \cap C_{p} \times R_{f(p)}\right) \cap L_{\xi_{p}+m(p)}: p=1,2, \cdots\right\} .
\end{aligned}
$$

Further, we obviously can consider that metric $\rho$ in a space $R_{\alpha+n}=$ $R_{\alpha} \times R_{n}$ is defined by the equality

$$
\rho\left((x, y),\left(x^{\prime}, y^{\prime}\right)\right)=\rho_{1}\left(x, x^{\prime}\right)+\rho_{2}\left(y, y^{\prime}\right), x, x^{\prime} \in R_{\alpha}, y, y^{\prime} \in R_{n},
$$

where $\rho_{1}$ and $\rho_{2}$ are metrics in $R_{\alpha}$ and $R_{n}$ respectively. Therefore, by virtue of the equality $F r V_{k} \times R_{\alpha} \cap C_{p} \times R_{f(p)}=\left(F r V_{k} \cap C_{p}\right) \times$ $R_{f(p)}$ we have 


$$
\begin{gathered}
d_{1}\left(\left(F r V_{k} \times R_{\alpha} \cap C_{p} \times R_{f(p)}\right) \cap L_{\varepsilon_{p}+m(p)}\right) \leqq d_{1}\left(F r V_{k} \times R_{\alpha} \cap C_{p} \times R_{f(p)}\right) \\
\leqq \\
d_{1}\left(F r V_{k} \cap C_{p}\right)+\operatorname{diam} R_{f(p)} .
\end{gathered}
$$

Since $R_{\alpha}=\omega\left(p_{\alpha}, R_{r}: \gamma \in \Gamma_{\alpha}\right)$, we have by Lemma $4.0 \lim _{p \rightarrow \infty} \operatorname{diam} R_{f(p)}=$ 0 , therefore by virtue of (5)

$$
\lim _{p \rightarrow \infty} d_{1}\left(\left(\boldsymbol{F r} V_{k} \times R_{\alpha} \cap C_{p} \times R_{f(p)}\right) \cap L_{\xi_{p}+m(p)}\right)=0 .
$$

Put

$$
\Theta_{p}=\left(F r V_{k} \times R_{\alpha} \cap C_{p} \times R_{f(p)}\right) \cap L_{\xi_{p}+m(p)} .
$$

By inductive assumption we have

$$
\text { ind } \Theta_{p} \leqq \text { ind } L_{\varepsilon_{p}+m(p)}=\psi\left(\xi_{p}+m(p)\right) .
$$

Moreover, by Lemma 1.1 (j)

$$
\sup \left\{\psi\left(\xi_{p}+m(p)\right): p=1,2, \cdots\right\} \leqq \psi(\alpha) .
$$

Since the sets $\Theta_{p}$ are open-closed and disjoint in

$$
M=\bar{R}_{n} \cup \cup\left\{\Theta_{p}: p=1,2, \cdots\right\},
$$

by virtue of (18), (19), (20), (21) and Lemma 3.4 we obtain

$$
\text { ind } M \leqq \psi(\alpha)
$$

By virtue of Lemma $1.1(\mathrm{j}) \psi(\alpha)<\psi(\alpha+m)$. Therefore, by virtue of (17), (23) $\operatorname{Fr} U \cap L_{\alpha+m} \subset M$ and inequality (16) holds. Thus, inequality (14) is proved. The proposition is completely proved.

Proof of Theorem 1.2. For any nonlimit number $\beta$ there exists such a number $\alpha+n(n \geqq 2)$ so that $\beta=\alpha+\bar{m}(n)$. We can merely put $\alpha+n=\beta+K(\beta)$. Therefore, by virtue of Proposition 5.1 there exists a compactum $X_{\beta}=L_{\alpha+\bar{m}(n)} \subset R_{\beta+K(\beta)}$ such that

$$
\text { Ind } X_{\beta}=\beta \text {, ind } X_{\beta}=\psi(\beta) \text {. }
$$

Since by Lemma $4.10 R_{\beta+K(\beta)}$ is weakly countable dimensional, $X_{\beta}$ is also weakly countable dimensional. Let $\beta_{0}$ be a limit number. Then we put

$$
X_{\beta_{0}}=\omega\left(p, X_{\beta}: \beta<\beta_{0}, K(\beta)>1\right) .
$$

Since $X_{\beta}$ are weakly countable dimensional, $X_{\beta_{0}}$ is also weakly countable dimensional. By virtue of Lemma 4.9 and (24) Ind $X_{\beta_{0}}=$ $\sup \left\{\right.$ Ind $\left.X_{\beta}=\beta: \beta<\beta_{0}, K(\beta)>1\right\}=\beta_{0}$

$$
\text { ind } X_{\beta_{0}}=\sup \left\{\text { ind } X_{\beta}=\psi(\beta): \beta<\beta_{0}, K(\beta)>1\right\} \text {. }
$$


By virtue of Lemma $1.1(\mathrm{~m})$ and (26) ind $X_{\beta_{0}}=\psi\left(\beta_{0}\right)$. Theorem 1.2 is completely proved. As it was mentioned in $\xi 1$ Theorem $1.2^{\prime}$ follows from Theorem 1.2. Therefore, Theorem $1.2^{\prime}$ is also proved.

From Proposition 5.1 and an equality (25) it follows that compactum $X_{\beta}$ imbeds in some compact $R_{\beta+K(\beta)} \in \rho_{\beta+K(\beta)}$ for nonlimit $\beta$, and $X_{\beta}$ imbeds in $R_{\beta}$ for limit $\beta$. Therefore the following assertion holds:

CoROLlary 5.1. For any $\beta<\omega_{1}$ there exists a compactum $X_{\beta}$ satisfying condition (24) and having an imbedding in some compact $R_{\beta+K(\beta)}$.

6. On small inductive dimension of product of spaces. This section is auxiliary. We shall prove here some results available for estimation of the small inductive dimension.

Definition 6.1. (See [6] Katetov). A mapping $f: X \rightarrow Y$ is called uniformly zero-dimensional if for any $\varepsilon>0$ there exists $\delta>0$ such that if the diameter of a set $M \subset Y$ is less than $\delta$, then $f^{-1}(M)$ is a union of a discrete collection of sets of the diameter < $\varepsilon$. We need the following assertions:

(K1) If $f: X \rightarrow Y$ is uniformly zero-dimensional mapping, then ind $Y \geqq$ ind $X$. (See [20], Zarelua.)

(K2) (See [6], Katetov.) $\operatorname{dim} X \leqq n$ if and only if there exists a uniformly zero-dimensional mapping $f: X \rightarrow I^{n}$ of the space $X$ into $n$-dimensional cube.

We also need the following theorem (see [18], Toulmin).

(T1) If a space $X$ has a dimension ind $X$ then ind $X \times I \leqq$ ind $X+I$ where $I=[0,1]$ is a segment.

LEMMA 6.1. ${ }^{12}$ Let $f: X \rightarrow Y$ be a uniformly zero-dimensional mapping and $g: X \times Z \rightarrow Y \times Z$ be a mapping defined by the equality $g(x, z)=f(x), z(x \in X, z \in Z)$. Then $g$ is also uniformly zero-dimensional.

The lemma is evident.

Proposition 6.1. For any finite dimensional space $X$ and for a space $Y$ having the dimension ind $Y$ the following inequality holds:

12 In what follows, we consider that on product $X \times Y$ of spaces $X, Y$ the metric is given by the following equality: $\rho\left((x, y),\left(x^{\prime}, y^{\prime}\right)\right)=\rho_{x}\left(x, x^{\prime}\right)+\rho_{y}\left(y, y^{\prime}\right),\left(x, x^{\prime} \in X, y\right.$, $\left.y^{\prime} \in Y\right), \rho_{x}, \rho_{y}$ are metrics in $X$ and $Y$ respectively. 


$$
\text { ind }(X \times Y) \leqq \text { ind } Y+\operatorname{dim} X \text {. }
$$

Proof. By Theorem (K2) there exists a uniformly zero-dimensional mapping $f: X \rightarrow I^{n}$, where $\operatorname{dim} X=n$. By Theorem (T1)

$$
\text { ind }\left(Y \times I^{n}\right) \leqq \text { ind } Y+n=\text { ind } Y+\operatorname{dim} X \text {. }
$$

Let $g: X \times Y \rightarrow I^{n} \times Y$ be a mapping defined by the equality

$$
g(x, y)=f(x), y \quad x \in X, y \in Y .
$$

Then, by virtue of Lemma $6.1 \mathrm{~g}$ is uniformly zero-dimensional mapping. From Theorem (K1) and inequality (2) follows (1).

Proposition 6.2. Let $\left\{U_{s}: s=1,2, \cdots\right\}$ be a collection of open sets in a space $X$ such that

$$
U_{s} \supset \bar{U}_{s+1}, U_{1}=X
$$

$$
\begin{gathered}
\lim _{s \rightarrow \infty} \operatorname{diam} U_{s}=0 \text { and } \cap\left\{U_{s}: s=1,2, \cdots\right\}=\{p\}, \\
\text { where } p \text { is a point in } X . \\
\text { ind }\left(U_{s} \backslash \bar{U}_{s+1}\right) \times I^{n} \leqq \alpha
\end{gathered}
$$

for some $n=1,2, \cdots, \alpha \geqq \omega_{0}$ and for any $s=1,2, \cdots$. Then for any space $K$ with $\operatorname{dim} K \leqq n$ the following inequality holds: ind $(X \times K) \leqq \alpha+[(n+3) / 2]$.

At first we need some preliminary lemmas. Let $(n+1)$ dimensional cube $I^{n+1}$ be a product of segments $[0,1]=I$. Then $I^{n+1}=$ $I \times I^{n}$. We denote by $\bar{I}^{n}$ the set $\{0\} \times I^{n} \in I^{n+1}$. We suppose that there is a collection of open sets $\mathscr{V}=\left\{V_{s}: s=1,2, \cdots\right\}$ in cube $I^{n+1}$ such that

$$
\begin{aligned}
& \text { The collection } \mathscr{T}_{1}=\left\{V_{s} \cap\left(I^{n+1} \mid \bar{I}^{n}\right): s=1,2, \cdots\right\} \\
& \text { is locally finite in }\left(I^{n+1} \mid \bar{I}^{n}\right) .
\end{aligned}
$$

The collection $\mathscr{T}_{2}=\left\{\boldsymbol{F r} V_{s}: s=1,2, \cdots\right\}$

is g.p. and $\operatorname{dim} \operatorname{Fr} V_{s} \leqq n$.

Then the following lemma holds:

Lemma 6.2. Let $(F, G)$ be a pair of disjoint closed sets in cube $I^{n+1}$ and

$$
\rho(F, G)>\sup \left\{\operatorname{diam} V_{s}: s=1,2, \cdots\right\}
$$


where $\rho(F, G)=\inf \{\rho(x, y): x \in F, y \in G\}, \rho$ is a metric in $I^{n+1}$. If $\mathscr{U}=\left\{U_{\mu}: \mu \in \mathscr{C}\right\}$ is a locally finite in $U=\left(I^{n+1} \mid \bar{I}^{n}\right)$ open covering of the set $U$ and $k=[(n+1) / 2]$, then there exists a partition $C$ in cube $I^{n+1}$ between $F$ and $G$ such that for any $s=1,2, \cdots$.

(9) The set $C \cap \operatorname{Fr} U_{s} \cap U$ is a union of $k$ closed sets $L^{1}, \cdots, L^{k}$ such that every set $L^{j}(j \leqq k)$ is a union of a discrete in $U$ countable collection $L^{j}$ of closed sets $L_{i}^{j}(i=1,2, \cdots)$ and every set $L_{i}^{j}$ is contained in some set $U_{\mu} \in \mathscr{W}$.

Proof. By virtue of (8) there exist open (in cube $I^{n+1}$ ) neighborhoods $O F$ and $O G$ of sets $F$ and $G$ respectively such that

$$
O F \cap O G=\phi \text {. }
$$

(11) Either $V_{s} \cap O F=\phi$ or $V_{s} \cap O G=\phi$ for any $V_{s} \in \mathscr{V}$.

Then, obviously

$$
\left.\left.\overline{\left(I^{n+1}\right.} \mid O F\right) \cap F=\dot{\phi}, \quad \overline{\left(I^{n+1}\right.} \backslash O G\right) \cap G=\phi .
$$

We note that by virtue of (6), (7) the collection $\mathscr{T}_{3}=\left\{F r V_{s} \cap U\right.$ : $s=1,2, \cdots\}$ is locally finite, and is g.p., and $\operatorname{dim} \operatorname{Fr} V_{s} \cap U \leqq n$. Therefore, by virtue of (10), (11) the conditions of Lemma 3.1 are satisfied for

$$
F_{1}=\overline{O F} \cap U, \quad G_{1}=\overline{O G} \cap U
$$

$$
D(n+1, k)=[(n+1) /([(n+1) / 2]+1)]=1, k=[(n+1) / 2], X=U \text {. }
$$

By Lemma 3.1 we obtain a partition $C^{\prime}$ in $U$ between $F_{1}$ and $G_{1}$ such that the condition (9) holds for $C=C^{\prime}$. (We note, that collection $\mathscr{L}^{j}$ is countable, because $U$ is separable.) For proving our lemma it is sufficient to show the existence of partition $C$ between $F$ and $G$ such that

$$
C^{\prime}=C \cap U \text {. }
$$

Since $C^{\prime}$ is a partition between $F$ and $G$ in $U$ there exist disjoint open sets $H_{1}$ and $H_{2}$ such that: $H_{1} \supset F_{1}, H_{2} \supset G_{1}, H_{1} \cup H_{2}=U \backslash C^{\prime}$, $H_{1} \cap H_{2}=\phi$. Then by virtue of (13) $H_{1} \subset U \backslash G_{1} \subset U \backslash \overline{O G} \subset I^{n+1} \mid \overline{O G}$. Similarly, $H_{2} \subset I^{n+1} \mid \overline{O F}$. Therefore by virtue of $(12) \bar{H}_{1} \cap G=\phi, \bar{H}_{2} \cap$ $F=\phi$. Since $I^{n+1}$ is hereditarily normal space, there exist open in $I^{n+1}$ sets $\widetilde{H}_{1}$, and $\widetilde{H}_{2}$ such that

$$
\widetilde{H}_{1} \supset H_{1} \cup F, \quad \widetilde{H}_{2} \supset H_{2} \cup F, \quad \widetilde{H}_{1} \cap \widetilde{H}_{2}=\phi
$$

therefore the set $C=I^{n+1} \backslash\left(\widetilde{H}_{1} \cup \widetilde{H}_{2}\right)$ is a partition between $F$ and $G$ and condition (14) holds. 
LEMMA 6.3. Let $f: X \rightarrow Y$ be a mapping and $Y=K \cup \cup\left\{L_{i}^{j}\right.$ : $i=1,2, \cdots, j=1, \cdots, k\}$. If the set $f^{-1}(K)$ is closed and finite dimensional, sets $L_{i}^{j}(i=1,2, \cdots)$ are open-closed in $K^{j}=K \cup \cup\left\{L_{i}^{j}\right.$ : $i=1,2, \cdots$ and $L_{i}^{j} \cap L_{i}^{j}=\phi$ for $i \neq i^{\prime}, L_{i}^{j} \cap K=\phi$ for any $i$. $\lim _{i \rightarrow \infty} \operatorname{diam} f^{-1}\left(L_{i}^{j}\right)=0$, ind $\left(X \backslash f^{-1}(K)\right) \leqq \alpha, \alpha \geqq \omega_{0}$ then ind $f^{-1}(y) \leqq$ $\alpha+(k-1)$.

Proof. Put $Z=f^{-1}(X)$, then obviously all conditions of Lemma 3.3 are satisfied. We have only to change notations. Consequently, our lemma follows from Lemma 3.3.

In this section we shall consider that a space $X$, satisfies the conditions of Proposition 6.2.

LEMmA 6.4. There is a mapping $f: X \times I^{n} \rightarrow I \times I^{n}=I^{n+1}$ such that:

(A) If for a sequence of sets $M_{i}, M_{i} \in I^{n+1} \lim _{i \rightarrow \infty} \operatorname{diam} M_{i}=0$, $\lim _{i \rightarrow \infty} \rho\left(\bar{I}^{n}, M_{i}\right)=0$, where $\rho\left(\bar{I}^{n}, M_{i}\right)=\inf \left\{\rho(x, y): x \in \bar{I}^{n}, y \in M_{i}, \rho\right.$ is a metric in $\left.I^{n+1}, \bar{I}^{n}=0 \times I^{n}\right\}$, then $\lim _{i \rightarrow \infty} \operatorname{diam} f^{-1}\left(M_{i}\right)=0$.

(B) For any point $x \in\{p\} \times I^{n} \subset X \times I^{n}$ and a closed set $F$, $x \notin F \subset X \times I^{n}$ we have:

$$
\overline{f(F)} \not \nexists f(x) .
$$

(C) The restriction of $f$ to $f^{-1}\left(\bar{I}^{n}\right)$ is a homeomorphism and $f^{-1}\left(\bar{I}^{n}\right)=\{p\} \times I^{n}$.

Proof. (A) Let us put $E=\{p\} \cup \cup\left\{F r U_{s}: s=1,2, \cdots\right\}$. Then $E$ is a closed subset of space $X$. We define a continuous function $g: E \rightarrow I$ by the equalities: $g(p)=0, g\left(F r U_{s}\right)=1 / s$. By Urysohn's theorem there is a continuous function $h: X \rightarrow I=[0,1]$ such that the restriction of $h$ to $E$ coincides with $g$ and if $x \in \bar{U}_{s} \mid U_{s+1}$ then $1 /(s+1) \leqq h(x) \leqq 1 / s$. We shall consider a continuous mapping $f$ : $X \times I^{n} \rightarrow I^{n}=I^{n+1}$ defined by the equality: $f(x, y)=h(x), y ; x \in X$, $y \in I^{n}$. Let $\pi_{1}: X \times I^{n} \rightarrow X, \pi_{2}: X \times I^{n} \rightarrow I^{n}, \pi_{3}: I \times I^{n} \rightarrow I^{n}, \pi_{4}: I \times$ $I^{n} \rightarrow I$ be projections. As it was mentioned above, we can consider $d\left((x, y), \quad\left(x^{\prime}, y^{\prime}\right)\right)=\rho_{X}\left(x, x^{\prime}\right)+\rho_{I^{n}}\left(y, y^{\prime}\right), x, x^{\prime} \in X, y, y^{\prime} \in I^{n}$, where $d$, $\rho_{X}, \rho_{I^{n}}$ are metrics in $X \times I^{n}, X, I^{n}$ respectively. Therefore, for proving (A) it is sufficient to show that

$$
\begin{aligned}
& \lim _{i \rightarrow \infty} \operatorname{diam} \pi_{1}\left(f^{-1}\left(M_{i}\right)\right)=0 \\
& \lim _{i \rightarrow \infty} \operatorname{diam} \pi_{2}\left(f^{-1}\left(M_{i}\right)\right)=0 .
\end{aligned}
$$

It is evident that if $\rho\left(\bar{I}^{n}, M_{i}\right)<1 / s$ then $\pi_{1} \circ f^{-1}\left(M_{i}\right)=h^{-1} \circ \pi_{4}\left(M_{i}\right) \subset U_{s}$. 
Consequently, by virtue of (4), equality (15) holds. Moreover, we obviously have $\pi_{2}\left(f^{-1}\left(M_{i}\right)\right)=\pi_{3}\left(M_{i}\right)$. Since $\lim _{i \rightarrow \infty} \operatorname{diam} M_{i}=0$ we obtain $\lim _{i \rightarrow \infty} \operatorname{diam} \pi_{3}\left(M_{i}\right)=0$. Therefore, equality (16) holds, and property (A) is proved.

(B) If $x \in p \times I^{n}, x \notin F$ then there is such a set $U_{s}$, and an open in $I^{n}$ set $V$ such that $x \in U_{s} \times V \subset X \times I^{n} \backslash F$, then obviously $f(x) \in[0,1 / s+1] \times V \subset I \times I^{n} \backslash f(F)$ and since $[0,1 /(s+1)] \times V$ contains an open neighborhood of a point $f(x)$ in $I^{n+1}$, property (B) holds. Property (C) is evident.

DEFINITION 6.2. (See [1] Borsuk). A covering $\mathscr{U}$ of an open set $U \subset Y$ is called canonical if for any point $x \in Y \backslash U$ and its any neighborhood $V$ there is a neighborhood $W \ni x$ in $Y$ such that if $G \in \mathscr{Q}$ and $G \cap W \neq \phi$ then $G \subset V$.

In [1] (Borsuk) it was proved that for any open set $U \subset Y$ there is an open canonical covering of $U$.

Lemma 6.5. Let $f: X \times I^{n} \rightarrow I^{n+1}$ be a mapping satisfying the condition of Lemma 6.4 and let in Lemma 6.2 a covering $U$ be canonical. Then, for the set $C$ satisfying the condition (9) we have ind $f^{-1}(C) \leqq \alpha+[(n+1) / 2]$.

Proof. By virtue of property (C) in Lemma 6.4 and by virtue of (4)

$$
\begin{aligned}
f^{-1}(C)= & \left(f^{-1}\left(\bar{I}^{n}\right) \cap f^{-1}(C)\right) \cup \cup\left\{f^{-1}(C)\right. \\
& \left.\cap\left(\left(U_{s} \mid \bar{U}_{s+2}\right) 2 \times I^{n}\right): s=1,2, \cdots\right\} .
\end{aligned}
$$

Since the sets $f^{-1}(C) \cap\left(\left(U \backslash \bar{U}_{s+2}\right) \times I^{n}\right)$ are open in $f^{-1}(C)$, then by virtue of (5) we have only to prove

$$
\operatorname{ind}_{x} f^{-1}(C) \leqq \alpha+[(n+1) / 2] \text { for } x \in f^{-1}(C) \cap f^{-1}\left(\bar{I}^{n}\right) .
$$

From Lemma 6.4 and condition $\left(8^{\prime}\right)$ it follows that the collection $\left\{f^{-1}\left(V_{s}\right): s=1,2, \cdots\right\}$ forms a basis in each point $x \in f^{-1}(C) \cap f^{-1}\left(\bar{I}^{n}\right)$. Since $\operatorname{Fr}\left(f^{-1}\left(V_{s}\right)\right) \subset f^{-1}\left(\operatorname{Fr} V_{s}\right)$ it is sufficient to prove that for any $s=1,2, \cdots$

$$
\text { ind } f^{-1}\left(\boldsymbol{F r} V_{s} \cap C\right)<\alpha+[(n+1) / 2] .
$$

If the condition (9) is satisfied and $U$ is canonical covering, then obviously $\lim _{i \rightarrow \infty} \operatorname{diam} L_{i}^{j}=0, \lim _{i \rightarrow \infty} \rho\left(\bar{I}^{n}, L_{i}^{j}\right)=0$ for $j \leqq k=[(n+1) /$ 2]. From Lemma 6.4 it follows that

$$
\lim _{i \rightarrow \infty} \operatorname{diam} f^{-1}\left(L_{i}^{j}\right)=0 \text {. }
$$

Put 


$$
K=\left(C \cap F r V_{s} \cap \bar{I}^{n}\right) .
$$

Then, obviously, $C \cap F r V_{s}=K \cup\left(C \cap F r V_{s} \cap U\right)$. From condition (9) it follows that

$$
C \cap F r V_{s}=K \cup \cup\left\{L_{i}^{j}: i=1,2, \cdots, j=1, \cdots, k\right\} .
$$

(23) Sets $L_{i}^{j}$ are open-closed in $K^{j}=K \cup \bigcup_{i=1}^{\infty} L_{i}^{j}$ and $L_{i}^{j} \cap L_{i}^{j}=\phi$ for $i \neq i^{\prime}$ and $K \cap L_{i}^{j}=\phi$ for any $i$.

From Lemma 6.4 (C) it follows that $\operatorname{dim} f^{-1}(K) \leqq n$. Moreover, since

$$
f^{-1}\left(C \cap \operatorname{Fr} V_{s} \cap U\right)=f^{-1}\left(C \cap F r V_{s}\right) \backslash f^{-1}(K) \subset X \times I^{n} \backslash\{p\} \times I^{n}
$$

we obtain by conditions (4), (5)

$$
\text { ind }\left(f^{-1}\left(C \cap F r V_{s}\right) \backslash f^{-1}(K)\right) \leqq \alpha .
$$

We put $X=f^{-1}\left(C \cap F r V_{s}\right), \quad Y=C \cap F r V_{s}$, then $f^{-1}(Y)=X$ and by virtue of conditions (20)-(24) and by Lemma 6.3 we have ind $f^{-1}(C \cap$ $\left.F r V_{s}\right) \leqq \alpha+(k-1)<\alpha+k$. Therefore, the inequality holds. The lemma is proved.

Lemma 6.6. There exists a collection of open sets $\mathscr{V}=\left\{V_{s}: s=\right.$ $1,2, \cdots\}$ in cube $I^{n+1}$ such that conditions (6), (7), (8') hold, and diam $V_{s}<\varepsilon$ for given $\varepsilon>0$.

Proof. By virtue of Corollary 2.2 there exists an open basis $A=\left\{U_{\alpha}: \alpha \in \mathscr{A}\right\}$ with boundaries of dimension $\operatorname{dim} \operatorname{Fr} U_{\alpha} \leqq n$ and a collection $\left\{\operatorname{Fr} U_{\alpha}: \alpha \in A\right\}$ is g.p. We can select for any $i=1,2, \cdots$ a finite covering $A_{i}$ of the set $\bar{I}^{n}$, consisting of elements of collection $A$ and satisfying the condition: $\operatorname{diam} T<\varepsilon / i$ for $T \in A_{i}$. Put $\mathscr{V}=\cup\left\{A_{i}: i=1,2, \cdots\right\}$. Then $\mathscr{V}$ obviously satisfies conditions (6), (7), $\left(8^{\prime}\right)$.

Lemma 6.7. ind $\left(X \times I^{n}\right) \leqq \alpha+[(n+3) / 2]$.

Proof. From conditions (4), (5) it follows that ind $\operatorname{lo}_{x}\left(X \times I^{n}\right) \leqq \alpha$ for any $x \in X \times I^{n} \backslash\{p\} \times I^{n}$. Therefore we have only to prove that

$$
\operatorname{ind}_{x}\left(X \times I^{n}\right) \leqq \alpha+[(n+3) / 2] \text { for } x \in\{p\} \times I^{n} .
$$

Let $f: X \times I^{n} \rightarrow I^{n+1}$ be a mapping, satisfying conditions $\mathrm{A}, \mathrm{B}, \mathrm{C}$ of Lemma 6.4 and let $x \in\{p\} \times I^{n}$ be any point. If $F$ is a closed set in $X \times I^{n} x \notin F$, then by virtue of (B) $\left.y=f(x) \notin \overline{f(F}\right)=G$. By virtue of one theorem in [1] Borsuk, there exists an open canonicla covering $\mathscr{C}$ of a set $U=I^{n+1} \mid \bar{I}^{n}$. By virtue of Lemma 6.6 there exists an 
open collection $\left\{V_{s}: s=1,2, \cdots\right\}$ satisfying the conditions (6), (7), $\left(8^{\prime}\right)$ and $\operatorname{diam} V_{s}<\varepsilon=\rho(f(x), \overline{f(F))}=\rho(y, G)$. Hence, the conditions of Lemma 6.2 hold and there is a partition $C$ in $I^{n+1}$ between $y$ and $G$, satisfying the condition (9). Then $f^{-1}(C)$ is obviously a partition between $x$ and $F$ in $X \times I^{n}$ and by Lemma 6.5

$$
\text { ind } f^{-1}(C) \leqq \alpha+[(n+1) / 2]<\alpha+[(n+3) / 2] \text {. }
$$

Consequently, inequality (25) holds.

Proof of proposition 6.2. By virtue of Theorem K2 there exists a uniformly zero-dimensional mapping $g: K \rightarrow I^{n}$. Let $r: X \times K \rightarrow$ $X \times I^{n}$ be a mapping defined by the equality $r(x, y)=x, g(y) x \in X$, $y \in K$. Then by Lemma $6.1 r$ is uniformly zero dimensional. Consequently, by Theorem $\mathrm{K} 1$ and Lemma 6.7 ind $(K \times X) \leqq$ ind $(X \times$ $\left.I^{n}\right) \leqq \alpha+[(n+3) / 2]$.

7. On dimensions of Smirnov's compacta. It is easy to show for a space $X=R_{\alpha}$, where $\alpha$ is a limit ordinal number, that all conditions of Proposition 6.2 are satisfied and, consequently, ind $R_{\alpha+n} \leqq \alpha+[(n+3) / 2]$. However, we can obtain more accurate estimation for ind $R_{\alpha+n}$.

Proposition 7.1. For a compact space $R_{\alpha+n}$ we have

$$
\text { ind } R_{\alpha+n} \leqq \alpha+[(n+2) / 2]
$$

where $\alpha$ is a limit number, $n=0,1,2, \cdots$.

Proof. Since for $n \leqq 2$ inequality (1) follows from Corollary 4.1, we can assume $n \geqq 3$. We consider a standard representation of the compactum $R_{\alpha+n} R_{\alpha+n}=\bar{R}_{n} \cup \cup\left\{R_{\gamma} \times R_{n}: \gamma \in \Gamma_{\alpha}\right\}$. It follows from Corollary 4.1 that

$$
\text { ind } R_{\gamma+n}=\text { ind } R_{\gamma} \times R_{n} \leqq \gamma+n<\alpha \quad\left(\gamma \in \Gamma_{\alpha}\right) .
$$

Since the set $R_{\gamma} \times R_{n}$ is open in $R_{\alpha+n}$ for any $\gamma \in \Gamma_{\alpha}$ we have only to prove that

$$
\operatorname{ind}_{x} R_{\alpha+n} \leqq \alpha+[(n+2) / 2] \text { for } x \in \bar{R}_{n} .
$$

Let $f: N \rightarrow \Gamma_{\alpha}$ be a bijection of the set of all integers $N$ onto $\Gamma_{\alpha}$. We put

$$
X_{p}=R_{\alpha} \backslash \cup\left\{R_{f(i)}: i=1,2, \cdots\right\} \quad(p \in N) .
$$

Let $\delta>0$ then we put 


$$
F_{1}=\bar{O}_{\delta}(x)^{13}, \quad F_{2}=\bar{R}_{n} \backslash O_{2 \delta}(x), \quad G=\bar{O}_{2 \delta}(x) \backslash O_{\delta}(x)
$$

By Corollary 2.2 there is an open basis $\mathscr{V}=\left\{V_{s}: s=1,2, \cdots\right\}$ in $\bar{R}_{n}$ such that $\mathscr{V}$ is a $\sigma$-locally finite collection (and since $\bar{R}_{n}$ is a compactum, the collection $\mathscr{V}$ is countable) and

$$
\begin{gathered}
\text { collection } \mathscr{V}_{1}=\left\{F r V_{s}: V_{s} \in \mathscr{V}, s=1,2, \cdots\right\} \\
\text { is g.p., } \operatorname{dim} \operatorname{Fr} V_{s} \leqq n-1 .
\end{gathered}
$$

We can obviously consider that

$$
\text { either } \bar{V}_{s} \cap F_{1}=\phi \text { or } \bar{V}_{s} \cap F_{2}=\phi \quad(s \in N) \text {. }
$$

Let $k=[n / 2], \quad \varepsilon=1 / p, \quad D(n, k)=[n /([n / 2]+1)]=1$ and $S_{p}=\left\{V_{s}\right.$ : $s \leqq p\}$ be a subcollection of a collection $\mathscr{V}$. Then by virtue of (6), (7), the conditions of Lemma 3.1 are satisfied and by this lemma there exists a partition $C_{p}$ in $\bar{R}_{n}$ between $F_{1}$ and $F_{2}$ such that

$$
d_{k}\left(C_{p} \cap \operatorname{Fr} V_{l}\right)<1 / p \quad l=1, \cdots, p .
$$

Let $q \in N$, then we set

$$
D=D(\delta, q, x)=G \cup \cup\left\{R_{f(p)} \times C_{p}^{\prime}: p=q, q+1, \cdots\right\} \subset R_{\alpha+n}
$$

where $C_{p}^{\prime}$ is an image of $C_{p}$ under the homeomorphism: $i: \bar{R}_{n} \rightarrow R_{n}$. Then $D$ is obviously a compactum. Let us show that

$$
\text { ind } D \leqq \alpha+[n / 2] \text {. }
$$

By virtue of (2) it is sufficient to prove that

$$
\operatorname{ind}_{x} D \leqq \alpha+[n / 2] \text { for } x \in G \text {. }
$$

We consider a collection of open sets $A=\left\{X_{p} \times V_{p}^{\prime}: p=1,2, \cdots\right\}$ $\left(V_{p}^{\prime}=i\left(V_{p}\right)\right.$ ) where $X_{p}$ is defined by (4). Then, obviously, $A$ forms a base in points of the compactum $\bar{R}_{n}$ and, consequently, in points $G \subset \bar{R}_{n}$. Therefore we have only to prove that

$$
\text { ind }\left(\boldsymbol{F r}\left(X_{l} \times V_{l}^{\prime}\right) \cap D\right) \leqq \alpha+([n / 2]-1) .
$$

It follows from (8), (9) that

$$
\begin{gathered}
\left(F r X_{l} \times V_{l}^{\prime}\right) \cap D \subset \bar{R}_{n} \cup \cup\left\{R_{f(p)} \times\left(F r V_{l}^{\prime} \cap C_{p}^{\prime}\right): p=q, q+1, \cdots\right\} \\
d_{k}\left(F r V_{l}^{\prime} \times C_{p}^{\prime}\right) \leqq 1 / p \quad l \leqq p
\end{gathered}
$$

Moreover, from Definition 4.3 and Lemma 4.0 it follows that

$$
\lim _{p \rightarrow \infty} \operatorname{diam} R_{f(p)}=0 \text {. }
$$

\footnotetext{
${ }^{13}$ Here by $O_{\delta}(x)$ we mean a $\delta$-neighborhood of $x$ in $\bar{R}_{n}(\varepsilon=\delta, 2 \delta)$.
} 
Consequently, from (14), (15) it follows that

$$
\lim _{p \rightarrow \infty} d_{k}\left(R_{f(p)} \times\left(F r V_{\imath}^{\prime} \cap C_{p}^{\prime}\right)\right)=0 .
$$

Since $R_{f(p)} \times\left(F r V_{l}^{\prime} \cap C_{p}^{\prime}\right) \subset R_{f(p)} \times R_{n}$, from condition (2) it follows that

$$
\sup \left\{\text { ind } R_{f(p)} \times\left(F r V_{l}^{\prime} \cap C_{p}^{\prime}\right): p=q, q+1, \cdots\right\} \leqq \alpha .
$$

From condition (13), (16), (17) and Lemma 3.4 it follows that

$$
\begin{gathered}
\text { ind }\left(F r\left(X_{l} \times V_{l}^{\prime}\right)\right) \cap D<\text { ind } \bar{R}_{n} \cup \cup\left\{R_{f(p)} \times\left(F r V_{l}^{\prime} \cap C_{p}^{\prime}\right): p\right. \\
=q, q+1, \cdots\} \leqq \alpha+(k-1)=\alpha+[n / 2]-1
\end{gathered}
$$

Thus, inequality (12) holds; consequently inequalities (11), (10) also hold. From construction of the set $D=D(\delta, q, x)$ it follows that

for any closed set $F$ and a point $x \in \bar{R}_{n}$ there exists a $\delta>0$ (18) and $q=1,2, \cdots$, such that the set $D(\delta, q, x)$ is a partition between $F$ and $x$.

From conditions (10), (18) follows (3) and, consequently (1).

Definition 7.1. (See [17] Smirnov). For any ordinal number $\beta<\omega_{1}$, we shall define a compactum $K_{\beta}$. For $\beta<\omega_{0} K_{\beta}$ is a $\beta$ dimensional cube. If $\beta$ is a limit number we put $K_{\beta}=\omega\left(p_{\beta} ; K_{r}\right.$ : $\gamma<\beta$ ). If $\beta=\alpha+n, \alpha=J(\beta), n=K(\beta)>0$ we put $K_{\beta}=K_{\alpha} \times I^{n}$.

It is evident that $K_{\beta} \in \rho_{\beta}$ (see Definition 4.3). In what follows $K_{\beta}$ will denote a compactum defined above. In [17] (Smirnov) it was proved that

$$
\text { Ind } K_{\beta}=\beta
$$

however, the equality

$$
\text { ind } K_{\beta}=\text { Ind } K_{\beta} \text {. }
$$

From Proposition 7.1 it follows that for $K(\beta) \geqq 3, \beta \geqq \omega_{0}$ equality is not true $(20)$ is false. However, for some $\beta$ it is true.

THEOREM 7.1. If $n=3,4, \cdots \alpha$ is a limit number $<\omega_{1}$, then for compactum $K_{\alpha+n}$, we have

$$
\text { ind } K_{\alpha+n} \leqq \alpha+[(n+2) / 2]<\alpha+n=\text { Ind } K_{\alpha+n} .
$$

If $\alpha$ is an invariant ordinal number then for $i=0,1,2$,

$$
\text { ind } K_{\alpha+i}=\text { Ind } K_{\alpha+i}=\alpha+i
$$


and besides that

$$
\text { ind } K_{\alpha+3}=\alpha+2 \text {. }
$$

Inequality (21) follows from inclusion $K_{\beta} \in \rho_{\beta}$ and Proposition 7.1. If $i=0,1$ then equality (22) follows from (19) and Corollary 1.2 (B), (C). Let us suppose that

$$
\text { ind } K_{\alpha+2} \geqq \alpha+2 \text {. }
$$

Since obviously $K_{\alpha+2}$ is topologically contained in $K_{\alpha+3}$ we have ind $K_{\alpha+3} \geqq \alpha+2$. The opposite inequality follows from condition (21). Since by virtue of (19) ind $K_{\alpha+2} \leqq$ Ind $K_{\alpha+2}=\alpha+2$, we have only to prove (24). To prove this inequality we need some preliminary results.

Definition 7.2. Let $X$ be a set in a product $E^{2} \times Y$ of a plane $E^{2}$ and an arbitrary space $Y$, and $p: E^{2} \rightarrow E^{2}$ be a reflection with respect to a straight line $\pi \subset E^{2}$. Let us consider a mapping $p(\pi)$ : $E^{2} \times Y \rightarrow E^{2} \times Y$, defined by the equality

$$
p(\pi)(x, y)=p(x), y \quad x \in E^{2}, y \in Y .
$$

The mapping $p(\pi)$ is called a reflection in $E^{2} \times Y$ with respect to $\pi$. The mapping $p(\pi)$ is obviously a homeomorphism, and $p(\pi)(c)=c$ for $c \in \pi \times Y$.

Definition 7.3. Let $X \subset E^{2} \times Y$ be an arbitrary set. We define $O(X, Y)$ as a minimal collection of sets in $E^{2} \times Y$ such that

(a) $X \in O(X, Y)$.

(b) If $Z \in O(X, Y)$ then for any reflection $q: E^{2} \times Y \rightarrow E^{2} \times Y$ we have $q(Z) \cup Z \in O(X, Y)$.

Lemma 7.1. Let $A, B$ be a pair of parallel straight lines in $E^{2}, \alpha$ be a limit number and $p_{\alpha}$ be an extra point in a compactum $K_{\alpha}=\omega\left(p_{\alpha}: K_{\beta}: \beta<\alpha\right)$. Let $M$ be a set in $E^{2} \times K_{\alpha}$ satisfying the condition

$$
\bar{M} \cap B \times K_{\alpha}=\phi .
$$

If the set $M$ contains a set $Q \times V$, where $Q$ is a square in $E^{2}$ such that one of its faces is contained in $A$, and $V \subset K_{\alpha}$, then for any compactum $K \subset A$ there exists a set $X \in O\left(M, K_{\alpha}\right)$ such that

$$
\bar{X} \cap B \times K_{\alpha}=\phi, \quad X \supset K \times V .
$$

Proof. Let $R$ be a rectangle in $E^{2}$ such that one of its faces 
is contained in $A$, and $\mu, \nu$ be a pair of two parallel straight lines containing two faces of $R$ which are perpendicular to $A$. Let $r_{\nu}\left(\tau_{\mu}\right): E^{2} \rightarrow E^{2}, p_{\nu}\left(p_{\mu}\right): E^{2} \times K_{\alpha} \rightarrow E^{2} \times K_{\alpha}$ be reflections in $E^{2}$ and in $E^{2} \times K_{\alpha}$ with respect to $\nu$ (respectively $\mu$ ). We put

$$
\begin{gathered}
T^{1}(R)=r_{\nu}\left(\left(r_{\mu}(R) \cup R\right)\right) \cup\left(r_{\mu}(R) \cup R\right), \\
L^{1}=(R \times V)=p_{\nu}\left(p_{\mu}(R \times V) \cup(R \times V)\right) \cup\left(p_{\mu}(R \times V) \cup(R \times V)\right) .
\end{gathered}
$$

Then obviously $L^{1}(R \times V)=T^{1}(R) \times V$ and the set $T^{1}(R)$ is also a rectangle such that one of its faces is contained in $A$. Therefore, sets $L^{1}\left(L^{1}(R \times V)\right), T^{1}\left(T^{1}(R)\right)$ are defined. We put

$$
\begin{aligned}
& L^{(n+1)}(R \times V)=L^{1}\left(L^{n}(R \times V)\right), \\
& T^{(n+1)}(R)=T^{1}\left(T^{n}(R)\right) .
\end{aligned}
$$

Therefore, the following condition holds

$$
A \subset \cup\left\{T^{n+1}(R), n=0,1, \cdots\right\}, \quad T^{n+1}(R) \times V=L^{n+1}(R \times V) .
$$

Consequently $A \times V \subset \cup L^{n+1}(R)$. Therefore, for any compactum $K \subset A$, there exists a number $n$ such that

$$
K \times V \subset L^{n}(R \times V) .
$$

Now let $R=O$. Let $O_{\perp}\left(M, K_{\alpha}\right)$ be a minimal collection of sets, satisfying the following conditions:

$$
M \in O_{\perp}\left(M, K_{\alpha}\right) \text {. }
$$

If $X \in O_{\perp}\left(M, K_{\alpha}\right), \pi \perp A$, then $(p(\pi)(X) \cup X) \in O_{\perp}\left(M, K_{\alpha}\right)$, where $p(\pi)$ is a reflection with respect to $\pi$. Then, obviously $O_{\perp}\left(M, K_{\alpha}\right) \subset O(M$, $\left.K_{\alpha}\right)$ and for any $n=1,2, \cdots$ there exists a set $X \in O_{\perp}\left(M, K_{\alpha}\right)$ such that $L^{n}(Q \times V) \subset X$. By virtue of (27) we have only to prove that

$$
\text { If } X \in O_{\perp}\left(M, K_{\alpha}\right) \text { then } \bar{X} \cap B \times K_{\alpha}=\phi \text {. }
$$

By virtue of (25) it is sufficient to prove that

(29) If $X \cap B \times K_{\alpha}=\phi, \mu \perp A$, then $\overline{P(\mu)(X) \cup X} \cap B \times K_{\alpha}=\phi$.

Since $\mu \perp A, A \| B$ and $P(\mu)$ is a homeomorphism, we have $P(\mu)(B \times$ $\left.K_{\alpha}\right)=B \times K_{\alpha}$,

$$
\phi=P(\mu)(\bar{X}) \cap P(\mu)\left(B \times K_{\alpha}\right)=P(\mu)(X) \cap B \times K_{\alpha} .
$$


property (29) and Lemma 7.1 are proved.

Lemma 7.2. Let $U$ be a subset in $E^{2} \times Y$. If $W \in O(U, Y)$ then there exists a set $L \in O(F r U, Y)$ such that $\operatorname{Fr} W \subset L$. 
Proof. Let $R: E^{2} \times Y \rightarrow E^{2} \times Y$ be a reflection, and $Z \subset E^{2} \times$ $Y$. Then since $R$ is homeomorphism, we have

$$
\operatorname{Fr}(Z \cup R(Z)) \subseteq \operatorname{Fr} Z \cup \operatorname{Fr} R(Z)=\operatorname{Fr} Z \cup R(F r Z) .
$$

Therefore, if $\operatorname{Fr} Z \subset L \in O(F r U, Y)$ then $\operatorname{Fr}(Z \cup R(Z)) \subset(L \cup R(L)) \epsilon$ $O(F r U, Y)$. Our lemma now follows from Definition 7.3.

CoROLlaRY 7.1. Let the conditions of Lemma 7.1 be satisfied, and $M$ be an open set. Then there exists an open set $X$ such that

$$
\operatorname{Fr} X \text { is a partition between } B \times K_{\alpha} \text { and } K \times V \text {. }
$$

There exists a set $C \in O\left(F r M, K_{\alpha}\right)$ containing $F r X$.

Proof. Since the set $M$ is open, the collection $O\left(M, K_{\alpha}\right)$ consists of open sets. By Lemma 7.1 there is a set $X \in O\left(M, K_{\alpha}\right)$ satisfying the condition (26). Since $X$ is open, the condition (30) holds. The property (31) follows from Lemma 7.2.

We shall use the following proposition proved in [18], (Toulmin).

(T2) Let $A, B$ be a pair of closed sets in a space $S, A \cup B=S$. If there is a homeomorphism $f: B \rightarrow A$ such that $f(c)=c$ for any $c \in A \cap B$ and dimension ind $S$ is defined, then ind $S=$ ind $A$.

LEMMA 7.3. Let $Z$ be an arbitrary set in a space $E^{2} \times Y$, then for any $X \in O(Z, Y)$ we have: ind $X=$ ind $Z$.

Proof. Since any reflection $R: E^{2} \times Y \rightarrow E^{2} \times Y$ is a homeomorphism and $R(c)=c$ for any point $c \in R(A) \cap A$ and any set $A \subset$ $E^{2} \times Y$, Lemma 7.3 follows from Theorem T2 and Definition 7.3.

Lemma 7.4. Let $(F, G)$ be a pair of opposite faces of a square $I^{2}$. Then there is a partition $C$ in compactum $I^{2} \times K_{\alpha}=K_{\alpha+2}$ between $F \times K_{\alpha}$ and $G \times K_{\alpha}$ such that

$$
\text { ind } C<\text { ind } K_{\alpha+2} \text {. }
$$

Proof. Since spaces $E^{2} \times K_{\alpha}$ and $K_{\alpha} \times I^{2}$ have imbeddings into each other

$$
\text { ind } K_{\alpha+2}=\text { ind } K_{\alpha} \times I^{2}=\text { ind } E^{2} \times K_{\alpha} \text {. }
$$

Let $\varphi: I^{2} \rightarrow E^{2}$ be a linear imbedding and $A, B$ be a pair of straight lines in $E^{2}$ such that $A \supset F, B \supset G$. We consider imbedding $\psi$ : $I^{2} \times K_{\alpha} \rightarrow E^{2} \times K_{\alpha}$ defined by the equality 


$$
\psi(x, y)=\varphi(x), y \quad\left(x \in I^{2}, y \in K_{\alpha}\right) .
$$

Now we can consider that $I^{2} \times K_{\alpha}$ is imbedded in $E^{2} \times K_{\alpha}$ by means of $\psi$. For any point $p \in F=F \times p_{\alpha}$ (where $p_{\alpha} \in K_{\alpha}$ is an extra point) there exists a neighborhood $M \ni p$ in $E^{2} \times K_{\alpha}$ such that

$$
\text { ind } \operatorname{Fr} M<\text { ind } E^{2} \times K_{\alpha} \quad M \cap B \times K_{\alpha}=\phi .
$$

Then there exist sets $V$ and $Q$ such that: $V$ is open in $K_{\alpha}, Q$ is a square in $E^{2}$, such that one of its faces is contained in $A, Q \times V \subset$ $M$ and

$$
V=K_{\alpha} \backslash \bigcup_{i=1}^{s} K_{\gamma_{i}} \quad\left(s=1,2, \cdots, \gamma_{i}<\alpha\right) .
$$

By virtue of Corollary 7.1 there exists a set $C^{1}$ such that

$$
\begin{gathered}
C^{1} \text { is a partition in } E^{2} \times K_{\alpha} \text { between } B \times K_{\alpha} \text { and } F \times V . \\
C^{1} \text { is contained in some set } R \in O\left(F r M, K_{\alpha}\right) .
\end{gathered}
$$

By virtue of Lemma 7.3 and conditions (34), (37) we have:

$$
\text { ind } C^{1} \leqq \text { ind } R=\text { ind } \operatorname{Fr} M<\text { ind } E^{2} \times K_{\alpha} \text {. }
$$

Let $T=\bigcup_{i=1}^{s} K_{r_{i}}=K_{\alpha} \backslash V$. Since obviously sets $E^{2} \times T$ and $I^{2} \times T$ have imbeddings into each other and $K_{r_{i}} \times I^{2}=K_{r_{i+2}}$, by virtue of (21), (35) we have ind $E^{2} \times T<\alpha$. Therefore for any partition $C^{2}$ in $E^{2} \times T$ between $B \times K \cap E^{2} \times T$ and $F \times T$

$$
\text { ind } C^{2}<\alpha \leqq \text { ind } E^{2} \times K_{\alpha} \text {. }
$$

We put $C^{3}=C^{1} \cup C^{2}$. Then by virtue of (36) the set $C^{3}$ contains a partition $C^{4}$ in $E^{2} \times K_{\alpha}$ between $B \times K_{\alpha}$ and $F \times K_{\alpha}$. Moreover,

$$
C^{1} \cap C^{2}=\phi \text {. }
$$

Consequently, by virtue of (38), (39), (40):

(41) $\quad$ ind $C^{4} \leqq$ ind $C^{3} \leqq \max \left(\right.$ ind $C^{1}$, ind $\left.C^{2}\right)<$ ind $E^{2} \times K_{\alpha}$.

We put $C=C^{4} \cap I^{2} \times K_{\alpha}$, then $C$ is a partition in $I^{2} \times K_{\alpha}$ between $F \times K_{\alpha}$ and $G \times K_{\alpha}=B \times K_{\alpha} \cap I^{2} \times K_{\alpha}$, and by virtue of (41), (33) ind $C \leqq$ ind $C^{4}<$ ind $E^{2} \times K_{\alpha}=$ ind $K_{\alpha+2}$.

Lemma 7.5. For any partition $C$ in $K_{\alpha+2}$ between $F \times K_{\alpha}$ and $G \times K_{\alpha}$ (where $(F, G)$ are opposite faces of $\left.I^{2}\right)$ we have ind $C \geqq \alpha+1$.

Proof. Since $K_{\alpha+2} \in \rho_{\alpha+2}$, by Lemma 4.8 Ind $C \geqq \alpha+1$. Let ind $C \leqq \alpha$. Since $\alpha$ is invariant number and by virtue of Theorem 
1.1 . Ind $C \leqq \varphi($ ind $C) \leqq \varphi(\alpha)=\alpha$. Consequently, ind $C \geqq \alpha+1$.

As we mentioned above, for proving Theorem 7.1 it is sufficient to prove inequality (24). However, this inequality directly follows from Lemmas 7.4, 7.5. Thus Theorem 7.1 is proved.

CoRollary 7.2. The equality ind $X \times I=$ ind $X+1$, where $I=$ $[0,1]$ is false even for a compact space $X$.

Proof. We put $X=K_{\omega_{0}+2}$. Since $\omega_{0}$ is an invariant number and $K_{\omega_{0}+3}=K_{\omega_{0}+2} \times I$ then by Theorem 7.1 ind $K_{\omega_{0}+2} \times I<$ ind $K_{\omega_{0}+2}+1$.

THEOREM 7.2. There exists a compactum $X$ such that for any finite dimensional separable space $Y$ with dimension ind $Y>0$ we have ind $X \times Y<$ ind $X+$ ind $Y$.

Proof. We put again $X=K_{\omega_{0}+2}$. Since $Y$ is separable space, ind $Y=\operatorname{dim} Y$ and by Theorem $\mathrm{K} 2(\S 6)$ there exists a uniformly zero-dimensional mapping $f: Y \rightarrow I^{n}$ where $n=$ ind $Y$. Let $g: K_{\omega_{0}+2} \times$ $Y \rightarrow K_{\omega_{0}+2} \times I^{n}=K_{\omega_{0}+n+2}$ be a mapping, defined by the equality $g(x$, $y)=x, f(y)$. Then by virtue of Lemma $6.1 \mathrm{~g}$ is a uniformly zerodimensional mapping. Consequently, by Theorem K1 $\S 6$ and by Theorem 7.1

$$
\text { ind } \begin{aligned}
X \times & Y=\text { ind } K_{\omega_{0}+2} \times Y \leqq \text { ind } K_{\omega_{0}+n+2} \leqq \omega_{0} \\
+ & {[(n+2) / 2]<\omega_{0}+n . }
\end{aligned}
$$

The last inequality holds because $n>0$.

8. On D-dimension. In [2] Henderson defined a transfinite $D$-dimension in the class of all metric $\operatorname{spaces}^{14}$ For any space $X$, $D(X)$ is either ordinal number or abstract symbol $4^{15}$.

Definition 8.1. We put $D(\phi)=-1$. If $X \neq \phi$ then $D(X)$ is the smallest ordinal number $\beta$ such that there exists a collection of sets $\left\{A_{\xi}: 0 \leqq \xi \leqq \gamma\right\}$ satisfying the following conditions:

(a) $X=\cup\left\{A_{\xi}: 0 \leqq \xi \leqq \gamma\right\}$.

(b) Every set $A_{\xi}$ is closed and finite dimensional.

(c) For any $\delta \leqq \gamma$ the set $\cup\left\{A_{\alpha}: \delta \leqq \alpha \leqq \gamma\right\}$ is closed in $X$.

(d) $J(\beta)=\gamma, \operatorname{dim} A_{\gamma} \leqq K(\beta)$.

(e) For any point $x \in X$ there exists the greatest number $\delta \leqq \gamma$

${ }^{14}$ Some results on $D$-dimension see also in [11], (Luxemburg).

${ }^{15}$ It is considered for every ordinal number $\beta$ that $\beta<\Delta, \Delta+\beta=\Delta$. 
such that $x \in A_{\dot{\delta}}$. If there is no such number $\beta$ we put $D(X)=\Delta$. If the conditions (a)-(e) hold then equality (a) is called a $\beta-D$-representation of a space $X$.

In [2] Henderson proved that for any compact space $X$ having the dimension Ind $X$ we have

(1) ind $X \leqq$ Ind $X \leqq D(X)$. $D(X)$.

(2) $|D(X)| \leqq$ weight $X$, where $|D(X)|$ is a cardinality of

(3) If $X$ is a finite dimensional space then $\operatorname{dim} X=D(X)=$ Ind $X$.

Proposition 8.1. (see also [12] (Luxemburg)). For any compactum $Y$ such that Ind $Y \geqq \omega_{0}, D(Y)<\Delta$ and for any ordinal number $\gamma, D(Y) \leqq \gamma<\omega_{1}$ there exists a compactum $Y_{r}$ containing $Y$ and satisfying the following condition

$$
D\left(Y_{r}\right)=\gamma, \text { Ind } Y_{r}=\text { Ind } Y \text {, ind } Y_{r}=\text { ind } Y \text {. }
$$

Proof. In [2] D. W. Henderson constructed for any $\gamma<\omega_{1}$ a compactum $X_{r}$ such that ind $X_{r}=$ Ind $X_{r}=\omega_{0}, D\left(X_{r}\right)=\gamma$. Let us put $Y_{r}=X_{r} \cup Y, Y \cap X_{r}=\phi$. Then, obviously $Y_{r}$ satisfies the conditions of Proposition 8.1.

COROLlaRY 8.1. There exist compacta $X$ such that ind $X<$ Ind $X<D(X)$.

Proof. By virtue of Theorem 1.2 there exists a weakly-countable dimensional compactum $X$ such that Ind $X>$ ind $X$. In [3] (Henderson) proved that every countable dimensional separable space $X$ has dimension $D(X)<\Delta$. From (2) it follows that $D(X)<\omega_{1}$. Consequently, Corollary 8.1 follows from Proposition 8.1.

THEOREM 8.1. If for a space $X, D(X)<\Delta$ then $X$ has dimension ind $X$ and

$$
\text { ind } X=\leqq D(X)+1^{16}
$$

LEMMA 8.1. If for any point $y \in Y$ there exists a neighborhood $O_{y} \ni y$ such that ind $O_{y}<\alpha$, then ind $Y \leqq \alpha$.

The lemma is evident.

${ }^{16}$ There is a mistake in [7] Kozlovsky, Proposition 11, where the author asserts the inequality ind $X \leqq D(X)$ in equivalent statement. 
LEMmA 8.2. Let (a) be a $\beta$-D-representation of a space $X, K(\beta)=$ $n>0$ and $C \subset X$ be a closed set such that

$$
\operatorname{dim} C \cap A_{r} \leqq n-1 .
$$

Then $D(C) \leqq J(\beta)+(n-1)$.

Proof. We put

$$
B_{\xi}=C \cap A_{\xi} \quad \xi \leqq \gamma .
$$

Then by virtue of (5)

$$
\operatorname{dim} B_{\gamma} \leqq(n-1)
$$

and

$$
C=\cup\left\{B_{\xi}: \xi \leqq \gamma\right\} \text {. }
$$

Since a) is a $\beta-D$-representation of $X$, from (6), (8), (7) it follows that $(8)$ is a $(\beta-1)-D$-representation of $C$.

Lemma 8.3. Let (a) be a $\beta$-D-representation of a space $X$ and $\gamma \geqq \omega_{0}$. We put

$$
U_{\delta}=X \backslash \cup\left\{A_{\xi}: \xi \geqq \delta: \delta<\gamma\right\} .
$$

Then the set $U_{\delta}$ is open and $D\left(U_{\delta}\right) \leqq \delta+s<\gamma$ for some $s=1,2 \cdots$.

Proof. Since (c) holds, $U_{\delta}$ is open. Let $\delta=T(\delta)+K(\delta)$. We put

$$
\begin{gathered}
B_{J(\hat{\delta})}=\cup\left\{A_{(J(\delta)+i)} \cap U_{\delta}: i=0, \cdots, K(\delta)\right\} \\
B_{\mu}=A_{\mu} \cap U_{\delta} \quad(\mu<J(\delta)) \\
s=\operatorname{dim} B_{J(\hat{o})} .
\end{gathered}
$$

Then

$$
U_{\tilde{o}}=U\left\{B_{\mu}: \mu \leqq J(\delta)=J(\delta+s)\right\} .
$$

Since (a) is a $\beta-D$-representation of $X$ and by virtue of (10), (11), (12), the equality (13) is a $(\delta+s)$-D-representation of $U_{j}$.

Proof of Theorem 8.1. We shall prove the theorem by induction on $\beta=D(X)$. If $D(X)<\omega_{0}$ then by virtue of (3)

$$
\text { Ind } X=D(X)=\operatorname{dim} X \geqq \text { ind } X \text {. }
$$

Let $D(X)=\beta \geqq \omega_{0}$ and (a) be a $\beta$-D-representation of $X$. Let us 
show that

$$
\text { ind }\left(X \backslash A_{\gamma}\right) \leqq \gamma=J(\beta) \text {. }
$$

Let $x \in X \backslash A_{T(\beta)}$, then by virtue of (e) there exists such $\delta_{0}<\gamma$ that $x \notin A_{\dot{o}}$, for $\delta^{\prime}>\delta_{0}$. Consequently,

$$
x \in U_{\delta_{0}+1}=X \backslash \cup\left\{A_{\delta^{\prime}}: \delta^{\prime} \geqq \delta_{0}+1 ; \delta^{\prime}<\gamma\right\} .
$$

By virtue of Lemma $8.3 D\left(U_{\tilde{o}_{0}+1}\right)<\gamma$. Consequently, by inductive assumption ind $U_{\delta_{0}+1} \leqq D\left(U_{\delta_{0}+1}\right)+1<\gamma$. Inequality (14) now follows from Lemma 8.1. Let $x \in X$ and $F \ni x$ be a closed set in $X$. If $K(\beta)=n>0$ then there is a partition $C$ between $x$ and $F$ such that inequality (5) holds. By virtue of Lemma 8.2 and by inductive assumption ind $C \leqq D(C)+1$. Consequently, inequality (4) holds. If $K(\beta)=0$ then by virtue of (d) $\operatorname{dim} A_{r} \leqq 0$ and we can find a partition $C$ between $x$ and $F$ such that $C \cap A_{r}=\varnothing$. Consequently, $C \subset X \backslash A_{\gamma}$. By virtue of (14) ind $C \leqq$ ind $\left(X \backslash A_{\gamma}\right) \leqq J(\beta) \leqq D(X)$. The theorem is proved.

Inequality in Theorem 8.1 cannot be improved. We give an example of a space $X$ such that

$$
\text { ind } X=D(X)+1 \text {. }
$$

(A) Construction. Let $I^{n} n=1,2, \cdots$ be a collection of cubes. We can consider that on each cube $I^{n}$ is defined a metric $\rho_{n}$ such that there exist two points $x_{n}, y_{n} \in I^{n}$ with $\rho_{n}\left(x_{n}, y_{n}\right)=1$. We identify all points $\left\{x_{n}\right\}$ in disjoint sum $\cup\left\{I^{n}: n=1,2, \cdots\right\}$ with the point $\{p\}=x_{1}$. We obtain the set $X=\bigcup_{n=1}^{\infty} I^{n}, I^{n} \cap I^{n^{\prime}}=\{p\}$ for $n \neq n^{\prime}$. In $X$ we define the following metric:

$$
\rho(x, y)=\left\{\begin{array}{l}
\rho_{n}(x, y) \text { for } x, y \in I^{n} \\
\rho_{n}(x, p)+\rho_{m}(y, p) \text { if } x \in I^{n}, y \in I^{m}, m \neq n .
\end{array}\right.
$$

LEMMA 8.4. ind $X=\omega_{0}+1$.

Proof. Let us show that

$$
\text { ind } X \geqq \omega_{0}+1 \text {. }
$$

We put $F=\bigcup_{n=1}^{\infty}\left\{y_{n}\right\}$. Then $F$ is a closed set in $X$ and $p \notin F$. Let $C$ be a partition between $\{p\}$ and $F$. Then $C \cap I^{n} \subset C$ is a partition between $\{p\}$ and $\left\{y_{n}\right\}$ in cube $I^{n}$. Consequently, for every $n$ ind $(C \cap$ $\left.I^{n}\right) \geqq(n-1)$ and inequality (17) holds. The inequality ind $X \leqq \omega_{0}+1$ follows from Theorem 8.1 and the following lemma.

Lemma 8.5. For any n-dimensional cube $I^{n} n=1,2, \cdots$ we 
have

$$
D\left(X \times I^{n}\right)=D(X)+n
$$

and $D(X)=\omega_{0}$.

Proof. The equality (18) holds if there is such a point $x \in X$ that $\sup \{D(O x): O x$ is a neighborhood of $x\}=D(X)$ (see [2]). For a point $p \in X$ and any neighborhood $O p \ni p$ we obviously have $D(O p) \geqq \omega_{0}$. Consequently, for proving our lemma we have only to show $D(X) \leqq \omega_{0}$. Let us put $A_{n}=I^{n}, A_{\omega_{0}}=\{p\}$. Then the equality $X=\left\{A_{\alpha}: \alpha \leqq \omega_{0}\right\}$ is clearly a $\omega_{0}-D$-representation $X$.

Thus, condition (15) is satisfied. The space $X$ has also some interesting property.

Proposition 8.2. For any n-dimensional space $K$

$$
\text { ind } X \times K \leqq \omega_{0}+[(n+3) / 2] \text {. }
$$

Thus, although $\omega_{0}=D(X)<$ ind $X=\omega_{0}+1$ but for $n \geqq 4$, by Lemma 8.5

$$
D\left(X \times I^{n}\right)=\omega_{0}+n>\omega_{0}+[(n+3) / 2] \geqq \text { ind }\left(X \times I^{n}\right) .
$$

Proof. Let $U_{s}$ be a $(1 / s)$ neighborhood of the point $p$. Since $X \backslash U_{s}$ is clearly a discrete union of finite dimensional sets, we have ind $\left(X \backslash U_{s}\right) \times I^{n} \leqq \omega_{0}$ for any $s=1,2, \cdots$. Consequently conditions (3), (4), (5) of Proposition 6.2 are satisfied and the inequality (19) holds.

THEOREM 8.2. Let $X$ be a compactum and $D(X)=\alpha+n$ where $\alpha \geqq \omega_{0}$ is a limit number and $n=0,1,2, \cdots$, then ind $X \leqq \alpha+$ $[(n+3) / 2]$.

CoRollary 8.2. If for a compactum space $X, D(X)=\beta+4, \beta \geqq$ $\omega_{0}$ then ind $X<D(X)$.

Proof. Let $\beta=\alpha+k, \alpha=J(\beta)$ then, by Theorem 8.2 ind $X \leqq$ $\alpha+[(k+7) / 2]=\alpha+[(k+1) / 2]+3<\alpha+k+4=\beta+4=D(X)$.

To prove Theorem 8.2 we need some preliminary lemmas.

LEMMA 8.6. Let $X$ be a compactum and the equality (a) be a $\beta$-D-representation of $X . \quad\left(\beta \geqq \omega_{0}\right)$, then for any neighborhood $O A_{\gamma}$ of the set $A_{r} D\left(X \backslash O A_{r}\right)<J(\beta)=\gamma$. 
Proof. By virtue of (e) the collection of sets $U_{\delta}, \delta<J(\beta)=\gamma$ defined by equality (9) is an open covering of $\left(X \backslash A_{r}\right)$. Since $X \backslash O A_{r}$ is a compactum, there exist such numbers $\delta_{1}, \cdots, \delta_{k},\left(\delta_{i}<\gamma\right)$ such that $X \backslash O A_{r}=\cup\left\{U_{\delta_{i}}: i=1, \cdots, k\right\}$. Let $\mu=\max \left\{\delta_{i}: i=1, \cdots,\right\}$. Since $\bigcup_{\delta^{\prime}} \subset U_{\tilde{o}}$ for $\delta>\delta^{\prime}$, we have $X \backslash O A_{r} \subset U_{\mu}$ and by virtue of Lemma 8.3 we have $D\left(X \backslash O_{\gamma}\right) \leqq D\left(U_{\mu}\right)<\gamma=J(\beta)$.

Let (a) be a $\beta$-D-representation of a compactum $X$, then we define a mapping

$$
\pi: X \longrightarrow X_{\sharp}
$$

by identifying all points of a set $A_{r}$. We put $\pi\left(A_{r}\right)=\rho$.

LEMma 8.7. The equality

$$
X_{\#}=\left\{B_{r}=\pi\left(A_{\xi}\right): \xi \leqq J(\beta)\right\}
$$

is a $J(\beta)$ D-representation of the compactum $X_{\#}$ and $B_{J(\beta)}=\{p\}$. Moreover, $\pi$ is a homeomorphism on the set $X \backslash A_{J(\beta)}$ and $\pi\left(X \backslash A_{J(\beta)}\right)=$

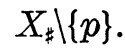

Lemma 8.7 is evidently follows from Definition 8.1 and the construction of mapping $\pi$.

LEMmA 8.8. Let $U$ be an open set in a space $X, A=X \backslash U$. If $f: X \rightarrow K, \quad g: X \rightarrow T$ are mappings such that $\operatorname{dim}\left(f^{-1}(x)\right)$ $\cap U \leqq 0, \operatorname{dim}\left(g^{-1}(y) \cap A\right) \leqq 0,(y \in T, x \in K)$ then the mapping $F$ : $X \rightarrow K \times T$ defined by the equality $F(x)=(f(x), g(x))$ is zero dimensional.

Proof. Let $a=(x, y)$ be a point in $K \times T, x \in K, y \in T$. Then $F^{-1}(a)=f^{-1}(x) \cap g^{-1}(y)$. Therefore

$$
\begin{array}{r}
F^{-1}(a)=f^{-1}(x) \cap g^{-1}(y) \cap(A \cup U)=\left(\left(f^{-1}(x) \cap U\right) \cap g^{-1}(y)\right) \\
\cup\left(\left(g^{-1}(y) \cap A\right) \cap f^{-1}(x)\right) \subset\left(f^{-1}(x) \cap U\right) \cup\left(g^{-1}(y) \cap A\right) .
\end{array}
$$

The set $U$ is open, and consequently is $F_{o}$ set. Further, sets $f^{-1}(x)$, $g^{-1}(x), A$ are closed, consequently by the sum theorem for dimension $\operatorname{dim}$ we have $\operatorname{dim}\left(f^{-1}(x) \cap U\right) \cup\left(g^{-1}(y) \cap A\right) \leqq 0$. Our Lemma now follows from (21).

LEMmA 8.9. Let $X$ be a compactum and (a) (Definition 8.1) be its $\beta$-D-representation. Then there exists a zero-dimensional mapping $F: X \rightarrow X_{\#} \times I^{n}$ where $n=K(\beta)$. 
Proof. By virtue of (d) (Definition 8.1) $\operatorname{dim} A_{J(\beta)} \leqq n$. Consequently, by Hurewicz's theorem [5] there exists a zero-dimensional mapping $\varphi: A_{J(\beta)} \rightarrow I^{n}$. Let $\psi: X \rightarrow I^{n}$ be any extension of mapping $\varphi$. We define the mapping $F: X \rightarrow X_{\sharp} \times I^{n}$ by the equality: $F(x)=$ $\pi(x), \psi(x)$. Since by Lemma $8.7 \pi$ is a homeomorphism on the set $X \backslash A_{J(\beta)}$, then by virtue of Lemma $8.8 F$ is zero-dimensional mapping.

Proof of Theorem 8.2. By virtue of Lemma 8.9 there exists a zero-dimensional mapping $F: X \rightarrow X_{\sharp} \times I^{n}$. Since $X$ and $X_{\sharp} \times I^{n}$ are compacta, zero-dimensional mapping $F$ does not lower dimension ind (see [20]) (Zarelua) and

$$
\text { ind } X \leqq \text { ind } X_{\sharp} \times I^{n} \text {. }
$$

By Lemma 8.7 the equality (22) is a $J(\beta)$ - $D$-representation of $X$ and $B_{J(\beta)}$ is a point $p$. Let $\left\{U_{s}: s=1,2, \cdots,\right\}$ be a collection of open in $X_{\sharp}$ sets such that

$$
\begin{gathered}
U_{s} \supset \bar{U}_{s+1}, \quad U_{1}=X \\
\lim _{s \rightarrow \infty} \operatorname{diam} U_{s}=0, \bigcap_{s=1}^{\infty} U_{s}=\{p\} .
\end{gathered}
$$

Then by Lemma 8.6

$$
D\left(U_{s} \backslash \bar{U}_{s+1}\right) \leqq D\left(X_{\sharp} \backslash U_{s+1}\right) \leqq \gamma_{1}<\gamma=J(\beta)
$$

for some ordinal number $\gamma_{1}<\gamma$. In [2] Henderson, Theorem 5 it was proved that $D(Z \times T) \leqq D(Z) \oplus D(T)$ where " $\bigoplus$ " denotes the natural sum of ordinal numbers. In particular $D\left(\left(U_{s} \backslash \bar{U}_{s+1}\right) \times I^{n}\right) \leqq$ $D\left(U_{s} \mid \bar{U}_{s+1}\right) \oplus D\left(I^{n}\right)=D\left(U_{s} \mid \bar{U}_{s+1}\right)+n=\gamma_{1}+n<J(\beta)$. The last inequality is true, because $J(\beta)$ is a limit number. Consequently, by Theorem 8.1

$$
\text { ind }\left(U_{s} \mid \bar{U}_{s+1}\right) \times I^{n} \leqq \gamma_{1}+n+1<J(\beta) .
$$

By virtue of (24), (25), (26) and Proposition 6.2 ind $\left(X_{\sharp} \times I^{n}\right) \leqq J(\beta)+$ $[(n+3) / 2]=\alpha+[(n+3) / 2]$. Our theorem now follows from inequality (23).

\section{REFERENCES}

1. K. Borsuk, Theory of retracts, Monografie Matematyczne, Tom 44 Panstwowe Wyddawnictwo Naukowe, Warsaw, 1967.

2. D. W. Henderson, D-dimension I. A new transfinite dimension, Pacific J. Math., 26 (1968), 91-107.

3. — D-dimension II. Separable spaces and compactifications, Pacific J. Math., 26 (1968), 109-113. 
4. W. Hurewicz and H. Wallman, Dimension theory, Princeton-London, Princeton Univ. Press-Oxford Univ. Press (1948), Princeton N.J. Mathematical series.

5. W. Hurewicz, Uber Abbildungen von eudlichdimensionalen Raumen auf Teihmengen Cartesischer Raume Sgb, Preuss. Acad. Wiss., 33 (1933), 754-768.

6. M. Katetov, On the dimension of nonseparable metric spaces, Czechoslovak. Math. J., 2N. 4. (77) (1952), 333-368 (Russian), English summary.

7. I. Kozlovsky, Two theorems on metric spaces, Dokl. Akad. Nauk. SSSR. 204 (1972), 784-787=Soviet Math. Dokl., 13 (1972), 743-747.

8. B. T. Levsenko, Spaces transfinite dimension, Matem. Sbornik, (N.S.) 67 (109:2), (1965), 255-266 (Russian).

9. L. A. Luxemburg, On compact spaces with noncoinciding transfinite dimensions, Dok1. Acad. Nauk. SSSR. Tom 212 (1973), N. 5, 1593-1597=Soviet Math. Dokl., 14 (1973), N. 6. 10. - On transfinite inductive dimensions, Soviet Math. Dokl., 14 (1973), N. 2 388-393=Dokl. Akad. Nauk. SSSR. Tom 209 (1973), N. 2. 295-298.

11. On transfinite D-dimension. Reports of the IV All Union Topological Conference. Tbilisi, 1972.

12. - On infinite dimensional spaces with transfinite dimension, Soviet Math. Dokl., 12 (1971), N. 4, 1272-1276=Dokl. Akad. Nauk. SSSR. Tom 199 (1971), N. 6., 1243-1246.

13. - On cohomological dimension, Soviet Math. Dokl., 16 (1975), N. 1, =Dokl. Akad. Nauk. SSSR. Tom 220 (1975), N.=Dokl. Acad. Nauk. SSSR. Tom 220 (1975), N. 6. 14. K. Morita, Normal families and dimension theory for metric spaces, Math. Ann., 128 (1954), N. 4, 350-362.

15. P. Roy, Failure of equivalence of dimensional concepts for metric spaces, Bull. Amer. Math. Soc., 68 (1962), 609-613.

16. Yu. Smirnov, On transfinite dimension, Matem. Sbornik, 58 (100) Nr. 4 (1962), 415-422 (Russian)

17. - On Universal spaces for certain classes of infinite dimensional spaces, Izv. Akad. Nauk. SSSR. Ser. Mat., 23 (1959), 185-196. English transl., Amer. Math. Soc., Transl., (2) 21 (1962), 21-33.

18. G. H. Toulmin, Shuffing ordinals and transfinite dimension, Poc. London. Math. Soc., (3) 4 (1954), 177-195.

19. N. B. Vedenisov, Uchenye Zapisky, Mosk. Gos. Univer. ser. Matem., 30 (1939), 131-140 (Russian).

20. A. V. Zarelua, The equality of dimensions and bicompact extensions, (Russian) Dokl. Akad. Nauk. SSSR. (1962) V. 144 N. 4, 713-716.

Received January 30, 1979 and in revised form April 17, 1979.

UNIVERSITY OF KENTUCKY

Lexington, KN 40506 


\title{
PACIFIC JOURNAL OF MATHEMATICS
}

\section{EDITORS}

\author{
DoNALD BABBITT (Managing Editor) \\ University of California \\ Los Angeles, CA 90024 \\ Hugo RossI \\ University of Utah \\ Salt Lake City, UT 84112 \\ C. C. MOORE and ANDrew OGG \\ University of California \\ Berkeley, CA 94720
}

J. DugundJI

Department of Mathematics

University of Southern California

Los Angeles, CA 90007

R. FINN and J. MILGRAM

Stanford University

Stanford, CA 94305

\section{ASSOCIATE EDITORS}
R. Arens
E. F. BeCKenbaCH
B. H. NeumanN
F. WOLF
K. YoSHIDA

\section{SUPPORTING INSTITUTIONS}

UNIVERSITY OF ARIZONA

UNIVERSITY OF BRITISH COLUMBIA

CALIFORNIA INSTITUTE OF TECHNOLOGY

UNIVERSITY OF CALIFORNIA

MONTANA STATE UNIVERSITY

UNIVERSITY OF NEVADA, RENO

NEW MEXICO STATE UNIVERSITY

OREGON STATE UNIVERSITY

\author{
UNIVERSITY OF OREGON \\ UNIVERSITY OF SOUTHERN CALIFORNIA \\ STANFORD UNIVERSITY \\ UNIVERSITY OF HAWAII \\ UNIVERSITY OF TOKYO \\ UNIVERSITY OF UTAH \\ WASHINGTON STATE UNIVERSITY \\ UNIVERSITY OF WASHINGTON
}

The Supporting Institutions listed above contribute to the cost of publication of this Journal, but they are not owners or publishers and have no responsibility for its content or policies.

Mathematical papers intended for publication in the Pacific Journal of Mathematics should be in typed form or offset-reproduced, (not dittoed), double spaced with large margins. Please do not use built up fractions in the text of the manuscript. However, you may use them in the displayed equations. Underline Greek letters in red, German in green, and script in blue. The first paragraph or two must be capable of being used separately as a synopsis of the entire paper. Please propose a heading for the odd numbered pages of less than 35 characters. Manuscripts, in triplicate, may be sent to any one of the editors. Please classify according to the scheme of Math. Reviews, Index to Vol. 39. Supply name and address of author to whom proofs should be sent. All other communications should be addressed to the managing editor, or Elaine Barth, University of California, Los Angeles, California, 90024.

50 reprints to each author are provided free for each article, only if page charges have been substantially paid. Additional copies may be obtained at cost in multiples of 50 .

The Pacific Journal of Mathematics is issued monthly as of January 1966. Regular subscription rate: $\$ 102.00$ a year $(6$ Vols., 12 issues). Special rate: $\$ 51.00$ a year to individual members of supporting institutions.

Subscriptions, orders for numbers issued in the last three calendar years, and changes of address shoud be sent to Pacific Journal of Mathematics, P.O. Box 969, Carmel Valley, CA 93924, U.S.A Old back numbers obtainable from Kraus Periodicals Co., Route 100, Millwood, NY 10546.

\section{PUBLISHED BY PACIFIC JOURNAL OF MATHEMATICS, A NON-PROFIT CORPORATION}

Printed at Kokusai Bunken Insatsusha (International Academic Printing Co., Ltd.). 8-8, 3-chome, Takadanobaba, Shinjuku-ku, Tokyo 160, Japan. 


\section{Pacific Journal of Mathematics}

Vol. 93, No. $2 \quad$ April, 1981

Ilya Eugene Blum and Srinivasa Swaminathan, Continuous selections and

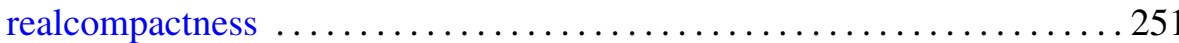

Lawrence James Brenton, Differentiably $k$-normal analytic spaces and extensions of holomorphic differential forms $\ldots \ldots \ldots \ldots \ldots \ldots \ldots 261$

Jo-Ann Deborah Cohen, Topologies on the ring of integers of a global field ................................................... 269

Robert Jay Daverman, Detecting the disjoint disks property $\ldots \ldots \ldots \ldots 277$

Edmund H. Feller, Rings where the annihilators of $\alpha$-critical modules are prime ideals ....................................... 299

Richard Elam Heisey and Henryk Torunczyk, On the topology of direct

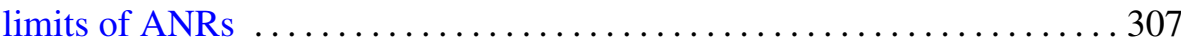

Gerald William Johnson and David Lee Skoug, Notes on the Feynman

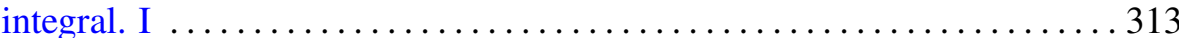

Michael S. Keane and Stuart Jay Sidney, Distinguishing a plane curve

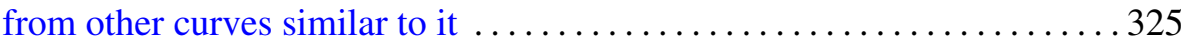

Leonid A. Luxemburg, On compact metric spaces with noncoinciding

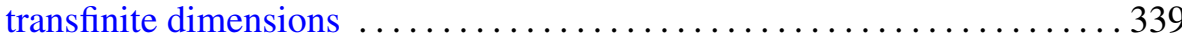

Chun Ming Ma, A uniqueness theorem for Navier-Stokes equations . . . . . . 387

Donald J. Newman and Theodore Joseph Rivlin, A characterization of the weights in a divided difference . . . . . . . . . . . . . . . . . . . 407

Marc Aristide Rieffel, $C^{*}$-algebras associated with irrational rotations $\ldots .415$

Kichi-Suke Saito, Invariant subspaces for finite maximal subdiagonal algebras

Frederic W. Shultz, Dual maps of Jordan homomorphisms and ${ }^{*}$-homomorphisms between $C^{*}$-algebras $\ldots \ldots \ldots \ldots$

Vsevolod Alekseevich Solonnikov, On the solvability of boundary and initial-boundary value problems for the Navier-Stokes system in domains with noncompact boundaries

Tavan Thomas Trent, New conditions for subnormality

L. E. Ward, Extending Whitney maps ..................

Leslie Wilson, Jets with regular zeros

Sergio Eduardo Zarantonello, The sheaf of $H^{p}$-functions in product 